\title{
Flora of Singapore precursors, 6: Typification of grass names relevant to the flora of Singapore
}

\author{
I.M. Turner ${ }^{1,2}$, D.J. Middleton ${ }^{2}$, H. Duistermaat ${ }^{3} \&$ J.F. Veldkamp ${ }^{3}$ \\ ${ }^{1}$ Singapore Botanical Liaison Officer, \\ Royal Botanic Gardens Kew, \\ Richmond, Surrey TW9 3AE, UK \\ i.turner@kew.org \\ ${ }^{2}$ Singapore Botanic Gardens, National Parks Board, \\ 1 Cluny Road, 259569 Singapore \\ ${ }^{3}$ Naturalis Biodiversity Center section Botany \\ P.O. Box 9517, 2300 RA, Leiden \\ The Netherlands
}

\begin{abstract}
The typification of more than 200 names of grass taxa relevant to the flora of Singapore is reviewed. A total of 52 lectotype designations are made here, including 22 which are posthumously published by the late J.F. Veldkamp. In addition, 13 second-step lectotypes (one by JFV), and two neotypes are designated. An earlier place of publication than generally cited for the combination Zizania latifolia is highlighted.
\end{abstract}

Keywords. Gramineae, lectotype, Poaceae, Singapore, typification

\section{Introduction}

In November 2017, Jan Frederik [Jan-Frits or JeF] Veldkamp died at the age of 76 (Baas \& Hovenkamp, 2018). For almost 50 years he had been the leading expert on the systematics of the grass family in the Malesian region. One of many projects in which he had agreed to collaborate was the Flora of Singapore, building on the work of Duistermaat (2005). Unfortunately, his death came before the family account for the Flora was completed, leaving a number of typifications for taxa included in the account unresolved. We have investigated these issues and present here a series of typifications. In some cases, lectotypes were indicated by Veldkamp in the unpublished manuscript and where we have found no reason to reject these choices (e.g. earlier typifications found or failure to locate the specimens indicated by Veldkamp) we include them here as lectotypes 'designated here by Veldkamp'. Veldkamp listed holotypes for many of the names in the manuscript. We have reviewed all such designations, as names validated before 1952 rarely have holotypes (McNeill, 2014), and have concluded that many of these either require lectotypification or have already been previously lectotypified.

When it is not entirely certain whether specimens are duplicates of a holotype or lectotype, but it is likely they are, these are indicated as 'possible isotype(s)' or 'possible isolectotype(s)'. The term 'fragment' is used here to indicate that the 
specimen is small or limited and probably a piece taken from another type specimen. Under the ICN (Turland et al., 2018) (Art. 8 Ex. 8) these fragments are to be treated as duplicates.

We include the basionyms or replaced synonyms of all names from the draft Flora of Singapore manuscript, either as the current name or as a synonym. The names are listed alphabetically with the accepted name indicated below if it differs from the basionym. Names now treated as synonyms are given in italics, accepted names in bold italics. Synonyms are not extensively cited here. Inclusion of taxa in the list should not be taken as making a definite statement on their presence as native or naturalised species in Singapore.

\section{List of grass names}

1. Acroceras sparsum Stapf ex Ridl., Fl. Malay Penins. 5: 229 (1925). - TYPE: Peninsular Malaysia, Selangor, Klang Gates below the ridge near the river, 2 January 1921, H.N. Ridley s.n. (lectotype K [K000290363], designated here).

= Acroceras tonkinense (Balansa) C.E.Hubb. ex Bor, Indian Forest Rec. 1: 78 (1938).

2. Agrostis diandra Retz., Observ. Bot. 5: 19 (1788). - TYPE: J. König s.n. [annot. 'Cinna diandra N.'] (lectotype LD [LD1215317], designated by Fischer, Bull. Misc. Inform. Kew 1932: 73 (1932); possible isolectotypes BM[2 sheets], C[5 sheets]).

= Sporobolus indicus (L.) R.Br. var. flaccidus (Roth) Veldkamp, Blumea 35: 433 (1991).

3. Agrostis elongata (R.Br.) Roth var. flaccida Roth in Roem. \& Schult., Syst. Veg. 2: 368 (1817). - TYPE: India orientali, Roth 80 (lectotype BM [BM000959534], designated by Baaijens \& Veldkamp, Blumea 35: 433 (1991); possible isolectotype LE [Herb. Trinius 1699.1]).

= Sporobolus indicus (L.) R.Br. var. flaccidus (Roth) Veldkamp, Blumea 35: 433 (1991).

4. Agrostis fertilis Steud., Syn. Pl. Glumac. 1: 170 (1854). - TYPE: Japan, Burger s.n. (lectotype L [L0050179], designated by Baaijens, Blumea 35: 437 (1991); possible isolectotypes L [L0050181], ? P).

= Sporobolus indicus (L.) R.Br. var. major (Buse) Baaijens, Blumea 35: 437 (1991).

5. Agrostis indica L., Sp. P1. 1: 63 (1753). - TYPE: [Clayton] 460-B (lectotype LINN [Herb. Linn. 84.36], designated by C.E. Hubbard, Agron. Lusit. 28: [67] (1967)).

= Sporobolus indicus (L.) R.Br., Prodr. 1: 170 (1810). 
6. Agrostis matrella L., Mant. P1. Altera 185 (1771). - TYPE: J.G. König 56 (lectotype LINN [Herb. Linn. 84.11], designated by Goudswaard, Blumea 26: 171 (1980); possible isolectotypes L [L0050867], LE [Herb. Trinius 396.1]).

= Zoysia matrella (L.) Merr., Philipp. J. Sci. 7: 230 (1912).

7. Agrostis maxima Roxb., Fl. Ind. 1: 319 (1820). - TYPE: W. Roxburgh s.n. (lectotype G [G00418718], designated here).

= Thysanolaena latifolia (Roxb. ex Hornem.) Honda, J. Fac. Sci. Univ. Tokyo, Sect. 3, Bot. 3: 312 (1930).

Notes. The lectotype specimen in Geneva has an original ticket in Roxburgh's hand saying Agrostis diandra with the epithet crossed out and replaced with maxima.

8. Agrostis procera Retz., Observ. Bot. 4: 19 (1786). - TYPE: J.G. König s.n. (lectotype LD [LD1215977], designated by Fischer, Bull. Misc. Inform. Kew 1932: 71 (1932); isolectotype BRI (fragment), K (fragment) [K000958820]).

= Eriochloa procera (Retz.) C.E.Hubb., Bull. Misc. Inform. Kew 1930: 256 (1930).

9. Aira indica L., Sp. Pl. 1: [1231] (1753). - TYPE: Sri Lanka, Sabaragamuwa Province, Ratnapura District, 11 miles E. of Daniyaya along Highway 17 to Ratnapura, near mile post 62, 22 October 1974, G. Davidse 7871 (neotype K [K000245262], designated by Renvoize in Cafferty et al., Taxon 49: 244 (2000); isoneotypes L, MO [MO-1410733]).

= Sacciolepis indica (L.) Chase, Proc. Biol. Soc. Washington 21: 8 (1908).

10. Andropogon L. sect. Amphilophis Trin., Mém. Acad. Imp. Sci. St.-Pétersbourg, Sér. 6, Sci. Math. 2: 285 (rank indicated p. 279) (1832). - TYPE: Andropogon laguroides DC. [= Bothriochloa laguroides (DC.) Herter] (lectotype designated here by Veldkamp).

11. Andropogon aciculatus Retz., Observ. Bot. 5: 22. (1789), as 'aciculatum'. - TYPE: J.G. König s.n. (lectotype LD [LD1224666], designated by Veldkamp, Austrobaileya 5: 509 (1999)).

= Chrysopogon aciculatus (Retz.) Trin., Fund. Agrost. 188 (1820). 
12. Andropogon affinis J.Presl in C.Pres1, Reliq. Haenk. 1: 343 (1830), nom. illeg., non R.Br. (1810). - Andropogon propinquus Kunth, Enum. P1. 1: 502 (1833). - TYPE: T.P.X. Haenke s.n. (lectotype PR [sheet no. 612301], designated here; isolectotypes PRC [PRC450151], US (fragment)).

= Sorghum propinquum (Kunth) Hitchc., Lingnan Sci. J. 7: 249 (1931).

13. Andropogon amaurus Buse, Gram. 20 (Feb 1854); Buse in Miq., Pl. Jungh. 3: 360 (Aug 1854). - TYPE: Java, H. Zollinger 539 (lectotype L [L0050026], designated by Veldkamp, Blumea 36: 180 (1991); isolectotypes G, K, P[3 sheets]).

= Polytrias indica (Houtt.) Veldkamp, Blumea 36: 180 (1991).

14. Andropogon annulatus Forssk., Fl. Aegypt.-Arab. 173 (1775), as 'annulatum'. - TYPE: Egypt, P. Forsskål 127 (lectotype C [C10001661], designated by Blake, Proc. Roy. Soc. Queensland 80: 65 (1969); isolectotype LD).

= Dichanthium annulatum (Forssk.) Stapf, F1. Trop. Afr. 9: 178 (1917).

15. Andropogon barbatus L., Mant. Pl. Altera 302 (1771), as 'barbatum', nom. illeg., non L. (1759). - TYPE: Anon. s.n. (lectotype LINN [Herb. Linn. 1211.21], designated by Judziewicz, Fl. Guianas, ser. A, Phanerogams 8: 136 (1990)).

= Chloris barbata Sw., F1. Ind. Occid. 1: 200 (1797).

16. Andropogon bladhii Retz., Observ. Bot. 2: 27 (1781). - TYPE: China, P.J. Bladh s.n. (lectotype LD [LD1218006], designated by Blake, Proc. Roy. Soc. Queensland 80: 62 (1969)).

= Bothriochloa bladhii (Retz.) S.T. Blake, Proc. Roy. Soc. Queensland 80: 62 (1969).

17. Andropogon brevifolius Sw., Prodr. 26 (1788). - TYPE: Jamaica, O. Swartz s.n. (lectotype M [M-0090295], designated by Hitchcock, Contr. U.S. Natl. Herb. 12: 143 (1908), excluding the portion which is Andropogon leucostachys; isolectotypes BM, $\mathrm{S})$.

= Schizachyrium brevifolium (Sw.) Nees in Mart., Fl. Bras. Enum. P1. 2: 332 (1829).

Notes. Hitchcock (1908) believed that there was no original material of this taxon in the Stockholm Herbarium and effectively selected material in Munich as the type. There is actually an extant Swartz specimen in Stockholm. 
18. Andropogon caricosus L., Sp. Pl., ed. 2, 2: 1480 (1763), as 'caricosum', nom. cons. - CONSERVED TYPE: Peninsula Ind. Orientalis, Herb. Wight Propr. No. 1687 (holotype K [K001050468]; possible isotype E).

= Dichanthium caricosum (L.) A.Camus, Bull. Mus. Natl. Hist. Nat. 27: 549 (1921).

19. Andropogon cerifer Hack., Fl. Bras. (Martius) 2(3): 281 (1883), as 'ceriferus'. - TYPE: Brazil, Rio de Janeiro, pr. Piqura, A.F.M. Glaziou 4296 (holotype W [W1904-0010930]; isotype US [US00156603] (fragment)).

= Cymbopogon citratus (DC.) Stapf, Bull. Misc. Inform. Kew 1906: 322 (1906).

20. Andropogon citratus DC., Cat. P1. Horti Monsp. 78 (1813), as ‘citratum'. - TYPE: Cult. in Horto Monspeliensis (not traced).

= Cymbopogon citratus (DC.) Stapf, Bull. Misc. Inform. Kew 1906: 322 (1906).

Notes: Stapf (1906: 322-335) provided a detailed history of lemon-grass and its taxonomy. He concluded that Andropogon citratus DC. was the oldest name available for the species and made the requisite combination in Cymbopogon.

21. Andropogon contortus L., Sp. P1. 2: 1045 (1753), as 'contortum'. - TYPE: [published illustration] 'Gram. Secalinum, Indicum, spica gracili, tomentoso, aristis longioribus, ad se invicem intortis', Plukenet, Phytogr. 4: t. 191, f. 5 (1692) (lectotype designated by Cope, Fl. Pakistan 143: 312 (1982)).

=Heteropogon contortus (L.) P. Beauv. ex Roem. \& Schult., Syst. Veg. 2: 836 (1817).

22. Andropogon crinitus Thunb. in Murray, Syst. Veg., ed. 14: 903 (1784), as 'crinitum'. - TYPE: Japan, C.P. Thunberg s.n. (lectotype UPS-THUNB [Herb. Thunberg 23880] [V-143127], designated here by Veldkamp).

= Pogonatherum crinitum (Thunb.) Kunth, Enum. P1. 1: 478 (1833).

23. Andropogon flexuosus Nees ex Steud., Syn. Pl. Glumac. 1: 388 (1854). - TYPE: $R$. Wight 1704 (lectotype E [E00174955], designated here; isolectotype LE [Herb. Trinius 204.1] (fragment)).

= Cymbopogon flexuosus (Nees ex Steud.) Will.Watson, Gaz. N. W. Ind. 10: 392 (1882).

Notes. In the protologue, Steudel refers to 'A. nardus Klein hrbr.' As Noltie (2005, p. 278) indicates, a sheet in $\mathrm{E}$ is annotated 'Andropogon nardus non est Hrb D. Rottler $\mathrm{Hb}$ Klein' indicating that this specimen came from Klein's herbarium. This is possibly not the sheet seen by Nees, which may have been among the wartime losses in Berlin, 
but it is likely to have the same provenance. As Noltie indicates, Wight's herbarium numbers relate to taxa rather than gatherings, so other Wight 1704 sheets cannot automatically be assumed to be true duplicates. There is however, a fragment from Nees's herbarium in Herb. Trinius.

24. Andropogon glaber Roxb., Fl. Ind. 1: 271 (1820). - TYPE: Bengal, W. Roxburgh s.n. (lectotype BM [BM000959769], designated by Simon, Austrobaileya 3: 79 (1989)).

= Bothriochloa bladhii (Retz.) S.T. Blake, Proc. Roy. Soc. Queensland 80: 62 (1969).

25. Andropogon intermedius R.Br., Prodr. 1: 202 (1810). - TYPE: Australia, Keppel Bay, R. Brown 6184 (lectotype BM [BM000939608], designated by Blake, Proc. Roy. Soc. Queensland 80: 62 (1969); isolectotypes E, F, K).

= Bothriochloa bladhii (Retz.) S.T. Blake, Proc. Roy. Soc. Queensland 80: 62 (1969).

26. Andropogon malacophyllus Hochst. ex Steud., Syn. Pl. Glumac. 1: 372 (1854).

- TYPE: India, Montibus Nilagiri, 1851, R.F. Hohenacker 917 (lectotype P [P02640887], designated here; isolectotypes CAL, CORD, FI, HAL, JE[2 sheets], K[2 sheets], L, LECB, MPU[2 sheets], S, TUB, W[3 sheets]).

= Ischaemum ciliare Retz., Observ. Bot. 6: 36 (1791).

27. Andropogon martini Roxb., Fl. Ind. 1: 277 (1820). - TYPE: W. Roxburgh s.n. (lectotype BM (lost), designated by Stapf, Bull. Misc. Inform. Kew 1906: 335-336 (1906)); India, Calcutta, Calcutta Botanic Garden, 18 February 1798, T. Hardwicke s.n. (neotype BM [BM000959800], designated here).

= Cymbopogon martini (Roxb.) Will.Watson, Gaz. N.-W. Ind. 10: 392 (1882).

Notes. Stapf (1906, pp. 335-336) reported two specimens of this species in BM, one from Roxburgh labelled Andropogon martini in his hand, and another collected by Hardwicke in the Calcutta Botanic Garden (in 1798 not 1789 as stated by Stapf). Stapf clearly referred to the Roxburgh sheet as type, so this represents an effective lectotypification. Unfortunately, this specimen seems now to be lost. While the Hardwicke collection is likely to be from the plants cultivated from the original seeds sent by Claude Martin to Roxburgh, the specimen shows no evidence of having been seen by Roxburgh and so is here designated as neotype rather than as a replacement lectotype. Note that Stapf excluded Roxburgh's drawing 1095 from A. martini and there does not appear to be any other original herbarium material available. 


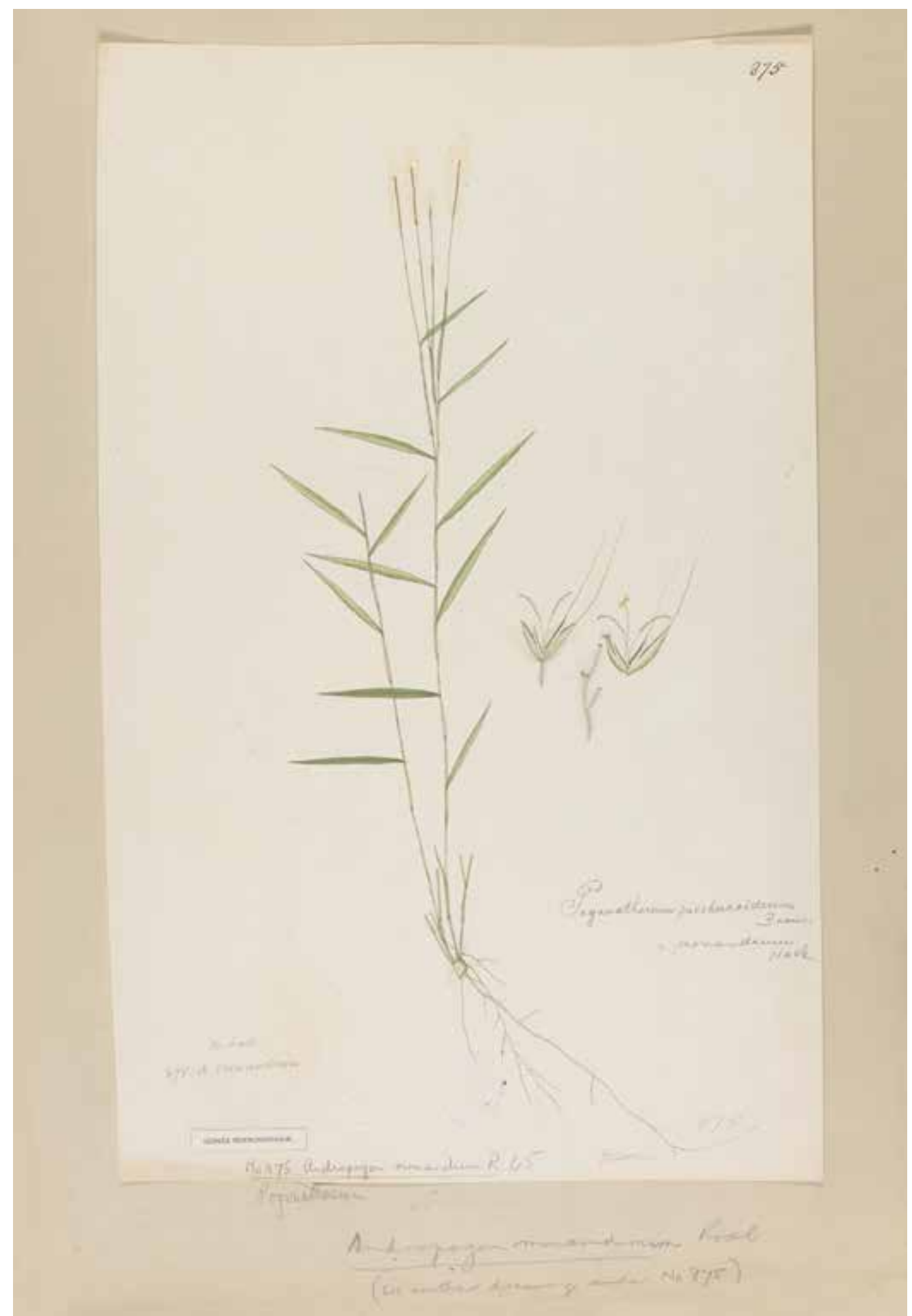

Fig. 1. Photograph of the lectotype of Andropogon monandrus Roxb., Icones Roxburghianae 875 in the Kew collection. Reproduced with the kind permission of the Director and the Board of Trustees, Royal Botanic Gardens, Kew. 
28. Andropogon monandrus Roxb., Fl. Ind. 1: 264 (1820). - TYPE: [unpublished illustration] Icones Roxburghianae 875 (lectotype K, designated here).

= Pogonatherum crinitum (Thunb.) Kunth, Enum. P1. 1: 478 (1833).

Notes. In the absence of any original herbarium material, Roxburgh's drawing (Fig. 1) is selected as lectotype.

29. Andropogon nardus L., Sp. P1. 2: 1046 (1753). - TYPE: Herb. Hermann 2: 66, No. 45 (lectotype BM [BM000594628], designated by Clayton \& Renvoize, Fl. Trop. E. Africa, Gram. (Part 3): 764 (1982)).

= Cymbopogon nardus (L.) Rendle in Hiern, Cat. Afr. P1. 2: 155 (1899).

30. Andropogon roxburghii Nees ex Wight \& Arn., Cat. Ind. Pl. (2): 100 (1834). - TYPE: non designatus.

= Cymbopogon citratus (DC.) Stapf, Bull. Misc. Inform. Kew 1906: 322 (1906).

Notes. McNeill in Noltie (2005, p. 278) confirmed that the name Andropogon roxburghii was validated in Wight's Catalogue by citation of Roxburgh's description of Andropogon schoenanthus L. while excluding the Linnaean type. A type for Andropogon roxburghii has yet to be selected from among the elements included in Andropogon schoenanthus by Roxburgh.

31. Anthistiria villosa Poir., Encycl., Suppl. 1: 396 (1810). - TYPE: Commerson s.n. (lectotype P-LA [P00563839], designated by Veldkamp, Blumea 61: 36 (2016)).

= Themeda villosa (Poir.) A.Camus, Fl. Indo-Chine 7: 364 (1922).

32. Anthoxanthum indicum L., Sp. P1. 1: 28 (1753). - TYPE: Herb. Hermann 1: 29, No. 25 (lectotype BM [BM000621332], designated Clayton, Fl. Trop. E. Africa, Gram. (Part 2): 395 (1974)).

=Perotis indica (L.) Kuntze, Revis. Gen. P1. 2: 787 (1891).

33. Aristida arundinacea L., Mant. P1. Altera 186 (1771). - TYPE: J.G. König s.n. (lectotype LINN [Herb. Linn. 98.8], designated by Hubbard, Fl. Trop. E. Africa, Gram. (Part 1): 133 (1970)).

= Neyraudia arundinacea (L.) Henrard, Meded. Rijks-Herb. $58: 8$ (1929).

34. Arundo donax L., Sp. P1. 1: 81 (1753). - TYPE: Anon. s.n. (lectotype L [Herb. A. van Royen No. 912.356-93], designated by Renvoize, Regnum Veg. 127: 21 (1993)). 
35. Arundo madagascariensis Kunth, Révis. Gramin. 2: 273, t. 48 (1830). - TYPE: Madagascar, L.M.A. du Petit-Thouars s.n. (lectotype P [P00446272], designated by Hubbard, Fl. Trop. E. Africa, Gram. (Part 1): 133 (1970); isolectotype US (fragment ex B)).

= Neyraudia arundinacea (L.) Henrard, Meded. Rijks-Herb. 58: 8 (1929).

36. Arundo reynaudiana Kunth, Révis. Gramin. 1: 275, t. 49 (1829). - TYPE: Burma, Pegu, 1829, A.A. Reynaud s.n. (lectotype B [B100365260], designated by Conert, Bot. Jahrb. Syst. 78: 240 (1959); isolectotypes L, LE [Herb. Trinius 2252.1] (fragment), P). = Neyraudia arundinacea (L.) Henrard var. zollingeri (Buse) Henrard, Blumea 3: 439 (1940).

37. Arundo versicolor Mill., Gard. Dict. ed. 8 no. 3 (1768). - TYPE: [published illustration] 'Arundo laconica versicolor, Park.', Morison, P1. Hist. Univ. 3: sect. 8, tab. 8, fig. 9 (1699) (lectotype designated here).

= Arundo donax L., Sp. Pl. 1: 81 (1753) var. versicolor (Mill.) Stokes, Bot. Mat. Med. 1: 160 (1812).

Notes. Miller did not cite any specimens but did refer to pre-Linnaean publications by Morison and Theophrastus. Morison's illustration (Fig. 2) is here designated lectotype for the variegated giant reed.

38. Arundo zollingeri Buse, Gram. 3 (Feb 1854); Buse in Miq., Pl. Jungh. 3: 343 (Aug 1854). - TYPE: Java, H. Zollinger 337 (lectotype L [L0044754], designated here by Veldkamp; isolectotypes G, K, L [L0044753], P).

= Neyraudia arundinacea (L.) Henrard var. zollingeri (Buse) Henrard, Blumea 3: 439 (1940).

39. Axonopus affinis Chase, J. Wash. Acad. Sci. 28: 180 (1938). - TYPE: USA, Mississipi, Waynesboro, 2 October 1896, T.H. Kearny 175 (holotype US [US00139602]; isotypes $\mathrm{GH}, \mathrm{MO}, \mathrm{US})$.

= Axonopus fissifolius (Raddi) Kuhlm., Relat. Commiss. Linhas Telegr. Estratég. Matto Grosso Amazonas 5(11): 87 (1922).

40. Cenchrus echinatus L., Sp. P1. 2: 1050 (1753). - TYPE: Anon. s.n. (lectotype L [Herb. A. van Royen No. 912.356-116], designated by Veldkamp, Regnum Veg. 127: 31 (1993)). 
41. Cenchrus granularis L., Mant. P1. Altera 575 (1771). - TYPE: Anon. s.n. (lectotype LINN [Herb. Linn. 1217.12], designated by Clayton \& Renvoize, F1. Trop. E. Africa, Gram. (Part 3): 849 (1982)).

= Mnesithea granularis (L.) de Koning \& Sosef, Blumea 31: 295 (1986).

42. Cenchrus inflexus R.Br., Prodr. 195 (1810), nom. illeg., non Poir. (1804). - TYPE: Australia, Arnheim South Bay, 6 February 1803, R. Brown 6140 (lectotype BM [BM000795713], designated by DeLisle, Iowa State Coll. J. Sci. 37: 280 (1963); isolectotypes E, K, P, US (fragment)).

= Cenchrus brownii Roem. \& Schult., Syst. Veg. 2: 258 (1817).

43. Cenchrus lappaceus L., Sp. P1., ed. 2, 2: 1488 (1763). - TYPE: Anon. s.n. (neotype LINN [Herb. Linn. 1212.15], designated by Monod de Froideville, Blumea 19: 59 (1971)).

= Centotheca lappacea (L.) Desv., Nouv. Bull. Sci. Soc. Philom. Paris 2: 189 (1810).

44. Cenchrus parviflorus Poir. in Lam., Encycl. 6: 52 (1804). - TYPE: Puerto Rico, E.P. Ventenat s.n. [?A. Riedlé leg.] (holotype P-LA [P00564021]; isotype US).

= Setaria parviflora (Poir.) Kerguélen, Lejeunia n.s. 120: 161 (1987).

45. Cenchrus setosus Sw., Prodr. 26 (1788). - TYPE: Jamaica, O.P. Swartz s.n. (lectotype S [S-R-969], designated by Hitchcock, Contr. U.S. Natl. Herb. 12: 143 (1908); possible isolectotypes BM, G, LD, S [2 sheets] SBT).

Notes. See notes under Panicum polystachion L. explaining why Cenchrus setosus is considered the accepted name for this species.

46. Chloris inflata Link, Enum. Hort. Berol. Alt. 1: 105 (1821). - TYPE: Germany, hort. bot. Berol. [sem. ex California], J.H.F. Link s.n. (lectotype B [B100248047], designated by Merrill, Enum. Philipp. Fl. Pl. 4: 241 (1926); isolectotype US (fragment).

= Chloris barbata Sw., F1. Ind. Occid. 1: 200 (1797).

47. Coix lacryma-jobi L., Sp. P1. 2: 972 (1753). - TYPE: Anon. s.n. (lectotype LINN [Herb. Linn. 1098.1], designated by Clayton \& Renvoize, Fl. Trop. E. Africa, Gram. (Part 3): 857 (1982)). 


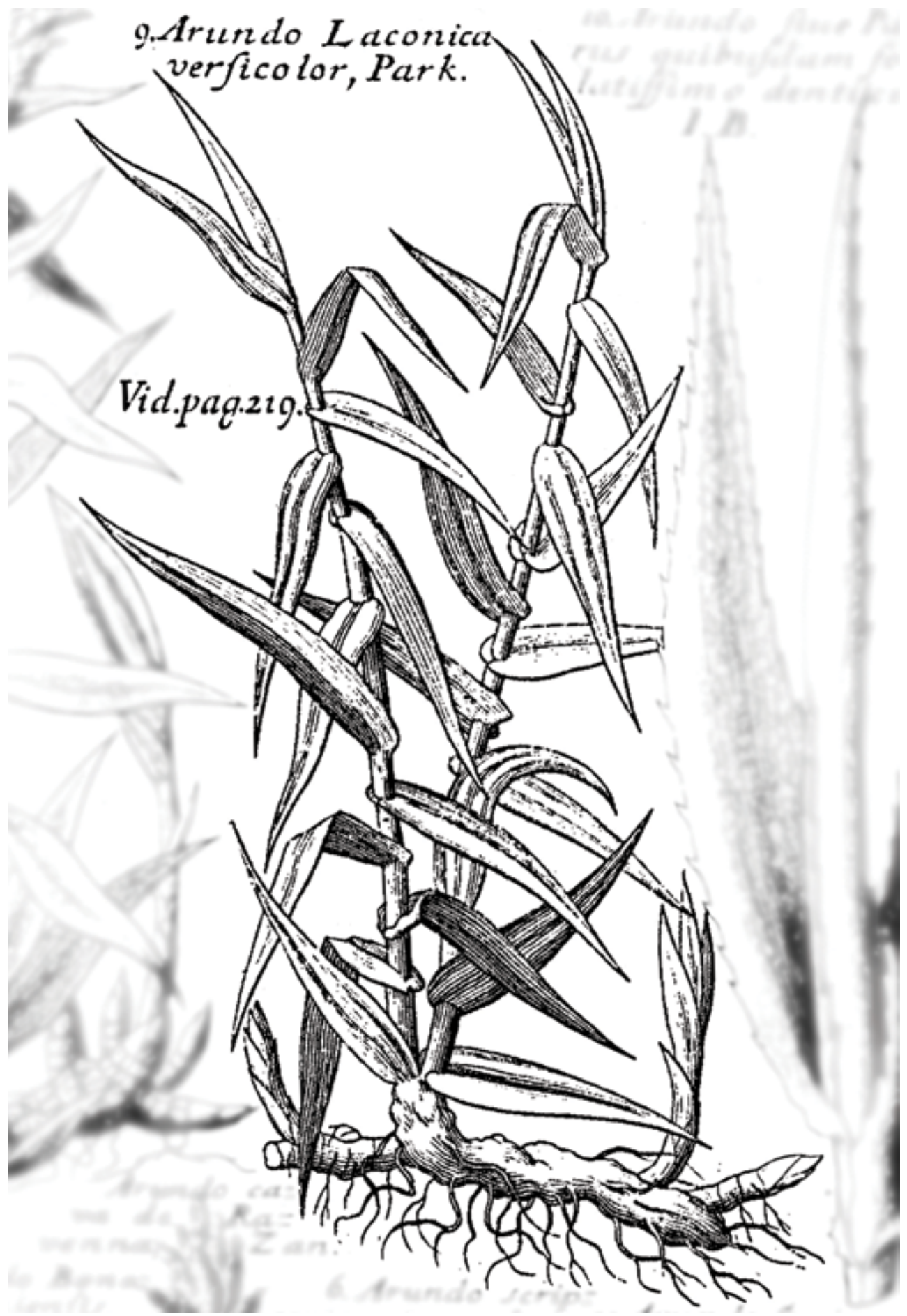

Fig. 2. Illustration of 'Arundo laconica versicolor', Park. Taken from Robert Morison's Plantarum Historiae Universalis Oxoniensis pars tertia, published in 1699. The surrounding figures have been faded using imaging software to avoid confusion. 
48. Coix ma-yuen Rom.Caill., Bull. Soc. Natl. Acclim. France, sér. 3, 8: 442 (1881), as 'ma-yu'én'. - TYPE: non designatus.

= Coix lacryma-jobi L. var. ma-yuen (Rom.Caill.) Stapf in Hooker, Fl. Brit. India 7: 100 (1896).

49. Cynodon parviglumis Ohwi, Bot. Mag. (Tokyo) 55: 538 (1941). - TYPE: Caroline Islands, [Federated States of Micronesia], Yap, 29 March 1938, R. Kanehira \& S. Hatusima 4348 (holotype FU; isotype US (fragment)).

= Cynodon dactylon (L.) Pers., Syn. P1. 1: 85 (1805).

50. Cynodon tener J.Presl in C.Presl, Reliq. Haenk. 1: 291 (1830). - TYPE: Philippines, T.P.X. Haenke s.n. (lectotype MO [MO-144078], designated by Lamson-Scribner, Rep. (Annual) Missouri Bot. Gard. 10: 36, 41 (1899); isolectotypes B, BM, HAL, LE [Herb. Trinius 2052.01], PR [sheet no. 495766], PRC, US (fragment), W[2 sheets]).

= Eustachys tenera (J.Presl) A.Camus, Rev. Int. Bot. Appl. Agric. Trop. 5: 208 (1925), as 'tener'.

51. Cynosurus aegyptius L., Sp. P1. 1: 72 (1753). - TYPE: Anon. s.n. (lectotype LINN [Herb. Linn. 91.11], designated by Kit Tan, Fl. Turkey 9: 578 (1985).

= Dactyloctenium aegyptium (L.) Willd., Enum. Pl. 1029 (1809), as 'aegyptiacum'.

52. Cynosurus coracanus L., Syst. Nat., ed. 10, 2: 875 (1759), as 'coracan'. - TYPE: [published illustration] Pluk., Phytographia 2: t. 91, f. 5 (1691) (lectotype designated by Phillips, Kew Bull. 27: 254 (1972)).

= Eleusine coracana (L.) Gaertn., Fruct. Sem. P1. 1: 8, t. 1, f. 11 (1788).

53. Cynosurus indicus L., Sp. P1. 1: 72 (1753). - TYPE: [published illustration] Burman, Thes. Zeyl. t. 47, f. 1. (1737) (lectotype designated by Phillips in Cafferty et al., Taxon 49: 249 (2000)). Sri Lanka, Kandy, 5 miles south of Matele on Kandy road, 16 January 2970, D. Clayton 5330 (epitype K [K000245128], designated by Phillips in Cafferty et al., Taxon 49: 249 (2000); isoepitypes CANB, PDA, US).

= Eleusine indica (L.) Gaertn., Fruct. Sem. P1. 1: 8 (1788). 
54. Digitaria caespitosa Ridl., Fl. Malay Penins. 5: 215 (1925). - TYPE: Singapore, Botanic Gardens, November 1920, H.N. Ridley s.n. (lectotype K [K00029074], first step designated by Veldkamp, Blumea 21: 64 (1973), second step designated here to exclude the material at the top of the sheet that Henrard has indicated as belonging to Digitaria marginata Link).

= Digitaria violascens Link, Hort. Berol. 1: 229 (1827).

55. Digitaria didactyla Willd., Enum. P1. 91 (1809). - TYPE: Réunion [Insula Borboniae], J.B. Bory de Saint-Vincent s.n. (lectotype B-W [B-W01646010], designated by Henrard, Monogr. Gen. Digitaria 179 (1950); isolectotype BM).

56. Digitaria elevatovenulosa Ohwi, Bull. Tokyo Sci. Mus. 18: 6 (1947). - TYPE: Singapore, 20 September 1941, J. Ohwi 6 (lectotype BO, designated by Henrard, Monogr. Gen. Digitaria 818 (1950); isolectotype L [L0044204]).

= Digitaria mollicoma (Kunth) Henrard, Blumea 1: 97 (1934).

57. Digitaria elevatovenulosa Ohwi var. glabra Ohwi, Bull. Tokyo Sci. Mus. 18: 6 (1947). - TYPE: Java, C.A. Backer 7318 (holotype BO).

= Digitaria mollicoma (Kunth) Henrard, Blumea 1: 97 (1934).

58. Digitaria fimbriata Link, Hort. Berol. 1: 226 (1827). - TYPE: Anon. s.n., p.p. (lectotype B [B100272564], designated by Henrard, Monogr. Gen. Digitaria 255 (1950), including part annotated ' $b$ ' only).

= Digitaria ciliaris (Retz.) Koeler, Descr. Gram. 27 (1802).

59. Digitaria marginata Link, Enum. Hort. Berol. Alt. 1: 102 (1821). - TYPE: Anon. s.n., p.p. (lectotype B [B200272564], designated by Henrard, Monogr. Gen. Digitaria 429 (1950), including part annotated 'a' only).

= Digitaria ciliaris (Retz.) Koeler, Descr. Gram. 27 (1802).

60. Digitaria microstachya Henrard, Monogr. Gen. Digitaria 454, fig. 942 (1950). - TYPE: Peninsular Malaysia, Malacca, Goldmines Clearings, W. Griffith s.n. ex Herb. Lehmann (holotype CGE [05576(CGE)]).

= Digitaria setigera Roth, Syst. Veg. 2: 474 (1817).

61. Digitaria nuda Schumach., Beskr. Guin. P1. 45 (1827). - TYPE: Guinea [Ghana], P. Thonning 367 (lectotype C [C10003772], designated by Henrard, Monogr. Gen. Digitaria 500 (1950); isolectotype L (fragment)). 
62. Digitaria peninsulae Ohwi, Bull. Tokyo Sci. Mus. 18: 7 (1947). - TYPE: Singapore, 20 September 1941, J. Ohwi 5 (lectotype BO, designated by Henrard, Monogr. Gen. Digitaria 538 (1950); isolectotype L [L0044144]).

= Digitaria didactyla Willd., Enum. P1. 91 (1809).

63. Digitaria pertenuis Buse, Gram. 41 (Feb 1854); Buse, in Miq., P1. Jungh. 3: 381 (Aug 1854). - TYPE: Sumatra, Padang, F.W. Junghuhn s.n. (lectotype L [L0044278], designated by Veldkamp, Blumea 21: 63 (1973); isolectotype U).

= Digitaria violascens Link, Hort. Berol. 1: 229 (1827).

64. Digitaria propinqua Gaudich., Voy. Uranie: 410 (1829), nom. illeg., non (R.Br.) P.Beauv. $(1812)=$ Panicum timorense Kunth, Enum. P1. 1: 83 (1833). - TYPE: Timor, Gaudichaud s.n. (lectotype P [P00898398], first step designated by Veldkamp, Blumea 21: 35 (1973), second step designated here; possible isolectotypes L, P [P00898396], US (fragm.)).

= Digitaria radicosa (J.Presl) Miq., Fl. Ned. Ind. 3: 437 (1857).

65. Digitaria setigera Roth, Syst. Veg. 2: 474 (1817). - TYPE: India, B. Heyne s.n. (lectotype B [B100272556], designated by Veldkamp, Blumea 21: 37 (1973); isolectotype B [B100272555]).

Notes. Veldkamp (1973) cited a Heyne specimen from Roth's herbarium in B as the holotype of Digitaria setigera. This is an effective lectotypification as he also referred to the Kew negative 2107 which is a picture of the sheet indicated as lectotype above. Bor (1955) had referred to the two sheets in B as the type.

66. Digitaria violascens Link, Hort. Berol. 1: 229 (1827). - TYPE: J.H.F. Link 93 (lectotype B [B100185495], designated by Henrard, Monogr. Gen. Digitaria 791 (1950); possible isolectotypes BAA (fragment), LE [Herb. Trinius 10009.3]).

67. Dimeria glabra Ridl., J. Fed. Malay States Mus. 10: 156 (1920). - TYPE: Singapore, Holland Road, edge of swampy hollow, 21 January 1919, I.H. Burkill SFN 4647 (lectotype K [K000290039], first step designated by Roberty, Boissiera 9: 398 (1960), second step designated here; isolectotypes G, K).

= Dimeria ornithopoda Trin., Fund. Agrost. 167 (1820).

68. Dimeria ornithopoda Trin., Fund. Agrost. 167, t. 14 (1820). - TYPE: India orientalis, Anon. s.n. (holotype LE [Herb. Trinius 1254.1]). 
69. Dimeria ornithopoda Trin. subvar. imperfecta Hack. in A.DC. \& C.DC., Monogr. Phan. 6: 82 (1889). - TYPE: Java, T. Lobb s.n. (holotype G).

= Dimeria ornithopoda Trin., Fund. Agrost. 167 (1820).

70. Eragrostis Wolf sect. Cataclastos Döll in Mart., Fl. Bras. 2(3): 137, 155 (1878). - TYPE: Eragrostis ciliaris (L.) R.Br. (designated here by Veldkamp).

71. Eragrostis cumingii Steud., Syn. P1. Glumac. 1: 266 (1854). - TYPE: Philippines, H. Cuming 1104 (lectotype P [P00622472], designated by Lazarides, Austral. Syst. Bot. 10: 109 (1997); isolectotypes BRI, CGE, G, E, GOET, K[2 sheets], L, MO, P, W).

72. Eragrostis malayana Stapf in Hook.f., Fl. Brit. India 7: 317 (1896). - TYPE: Peninsular Malaysia, Perak, Thaiping, L. Wray 773 (lectotype K [K000290370], designated by Veldkamp, Blumea 47: 180 (2002).

= Eragrostis montana Balansa, J. Bot. (Morot) 4: 168 (1890).

73. Eragrostis montana Balansa, J. Bot. (Morot) 4: 168 (1890). - TYPE: Cambodia, Mont de Pursat, 18 June 1875, A. Godefroy 487 (lectotype L [L0044358], designated here; isolectotype $\mathrm{P}[\mathrm{P} 02279651])$.

74. Eriachne pallescens R.Br., Prodr. 1: 184 (1810). - TYPE: New Holland [Australia], Endeavours River, 1770, J. Banks \& D. Solander s.n. (lectotype BM [BM000645842], designated by van Eck-Borsboom, Blumea 26: 134, 135 (1980); possible isolectotypes BM[4 sheets]).

75. Eriachne triseta Nees ex Steud., Syn. Pl. Glumac. 1: 237 (1854). - TYPE: Sri Lanka [Ceylon], Macrae s.n. [R. Wight 2047] (lectotype B [B100272753], designated here; isolectotype LE [Herb. Trinius 1844.1] (fragment)).

Notes. The protologue simply refers to Ceylon. A holotype in Paris was referred to by van Eck-Borsboom (1980, p. 136) without locating the specimen. This is presumably based on the assumption that the type was represented in Steudel's herbarium now in P. There is a specimen in Berlin that is annotated with the name in Nees's hand. This is selected as lectotype here. 
76. Eriochloa ramosa (Retz.) Kuntze var. involucrata Hack. ex Merr., Philip. J. Sci. 1, Suppl. 5: 349. (1906). - TYPE: Philippines, Mindoro, Puerta Galera, October 1903, E.D. Merrill 3333 (lectotype W [1916-0020483], designated here by Veldkamp; isolectotypes BM, K, NY, US).

= Eriochloa procera (Retz.) C.E.Hubb., Bull. Misc. Inform. Kew 1930: 256 (1930).

77. Graya elegans Nees ex Steud., Syn. P1. Glumac. 1: 119 (1854). - TYPE: Sri Lanka [Ceylon], R. Wight 2033 (lectotype LE [Herb. Trinius 691.1] (fragment), designated here).

= Sphaerocaryum malaccense (Trin.) Pilg., Feddes Repert. 45: 2 (1938).

Notes. There is a fragment from Herb. Nees in Herb. Trinius which is here designated as lectotype as Nees's specimens seem to have been lost.

78. Hemigymnia fusca Ridl., Fl. Malay Penins. 5: 228 (1925). - TYPE: Singapore, Chan Chu Kang, March 1890, H.N. Ridley 1704 (lectotype SING [SING0041601], designated here by Veldkamp; isolectotype K [K000290325]).

= Ottochloa nodosa (Kunth) Dandy, J. Bot. 69: 55 (1931).

79. Holcus bicolor L., Mant. P1. Altera 301 (1771). - TYPE: Herb. Clifford 468, Holcus 1 (BM [BM000647533], designated by Poilecot, Boissiera 56: 509 (1999)).

= Sorghum bicolor (L.) Moench, Methodus 207 (1794).

80. Holcus sorghum L., Sp. P1. 2: 1047 (1753). - TYPE: Herb. Clifford 468, Holcus 1 (BM [BM000647533], designated by Davidse in Cafferty et al., Taxon 49: 251 (2000)). = Sorghum bicolor (L.) Moench, Methodus 207 (1794).

81. Hydropyrum latifolium Griseb. in Ledeb., Fl. Ross. 4: 466 (1853). - TYPE: Russia, Amur, in lacubus inter Schilka et Argun, 1833, N.S. Turczaninov s.n. (lectotype LE, designated by Camus, Rev. Int. Bot. Appl. Agric. Trop. 30: 54 (1950); isolectotypes GOET, K[2 sheets], L, LECB, P, W[2 sheets], US).

= Zizania latifolia (Griseb.) Hance ex F.Muell., Proc. Acclim. Soc. Victoria 1: 419 (1872).

Note. Ferdinand von Mueller made the combination in Zizania for this species considerably earlier than Stapf, who is generally cited as the author of the combination. 
82. Imperata koenigii (Retz.) P. Beauv. var. maior Nees, F1. Afr. Austr. Ill. 90 (1841). - TYPE: South Africa, Facoskraal, 1900-2000', 10 February 1832, F.J. Drège 4253 (lectotype P [P00443973], designated here; isolectotypes K, L, TUB, W[2 sheets]). = Imperata cylindrica (L.) P. Beauv., Ess. Agrostogr. 165 (1812).

Notes. We presume that Nees's type was destroyed in Berlin, and therefore select as lectotype a sheet in the Paris Herbarium.

83. Isachne confusa Ohwi, Bull. Tokyo Sci. Mus. 18: 14 (1947). - TYPE: Indonesia, Banka, Muntok, 18 October 1917, H.A.B. Bünnemeijer 1577 (lectotype BO, designated by Jansen, Reinwardtia 2: 282 (1953); isolectotypes B, CAL, L[2 sheets], SING, US).

84. Isachne schmidtii Hack., Bot. Tidsskr. 24: 97 (1901). - TYPE: Thailand [Siam], Koh Chang Island, 1889-1900, J. Schmidt s.n. (lectotype W [1916-0021689], designated here, or possibly holotype; isolectotype US (fragment)).

85. Isachne semitalis Ridl., F1. Malay Penins. 5: 237 (1925). - TYPE: Singapore, Tanglin, March 1894, H.N. Ridley 6110 (lectotype SING [SING0017766], designated here by Veldkamp; isolectotype K [K000290172]).

= Isachne schmidtii Hack., Bot. Tidsskr. 24: 97 (1901).

86. Ischaemum apricum Ridl., Fl. Malay Penins. 5: 203 (1925). - TYPE: Peninsular Malaysia, Penang, Waterfall Gardens, 16 December 1918, I.H. Burkill SFN 4629 (lectotype K [K000290093], first step designated by Roberty, Boissiera 9: 332 (1960), second step designated here; isolectotypes ?G, SING [SING0219195], US).

= Ischaemum ciliare Retz., Obs. Bot. 6: 36 (1791).

87. Ischaemum aristatum L. var. imbricatum Hack. in A.DC. \& C.DC., Monogr. Phan. 6: 203 (1889). - TYPE: India, Khasia, Churra, June 1850, J.D. Hooker \& T. Thomson s.n. (lectotype K [K000245675], designated by Traiperm et al., Kew Bull. 67: 78 (2012); isolectotypes L, W).

= Ischaemum barbatum Retz., Observ. Bot. 6: 35 (1791).

88. Ischaemum barbatum Retz., Observ. Bot. 6: 35 (1791). - TYPE: Java, Anon. s.n. [?H.P. Wennerberg leg.] (holotype LD [1229226]). 
89. Ischaemum ciliare Retz., Obs. Bot. 6: 36 (1791). - TYPE: Anon s.n. [? P. Osbeck leg.] (holotype LD [1229166]).

90. Ischaemum commelynifolium Stapf ex Ridl., Fl. Malay Penins. 5: 202 (1925). - TYPE: Peninsular Malaysia, Kedah, Kedah Peak, June 1893, H.N. Ridley 5152 (lectotype K [K000290149], first step designated by Roberty, Boissiera 9: 333 (1960), second step designated here; isolectotypes G, SING).

= Ischaemum feildingianum Rendle, J. Bot. 32: 101 (1894).

91. Ischaemum feildingianum Rendle, J. Bot. 32: 101 (1894). - TYPE: Peninsular Malaysia, Malacca, Gunong Ledang (Mt Ophir), growing on rock of Padang Batu, November 1892, J.B. Feilding s.n. (lectotype BM [BM000959777], first step designated by Roberty, Boissiera 9: 333 (1960), second step designated here; isolectotypes E, G, K, SING).

Notes. The collector (and his eponymous epithets) are often mispelled as Fielding.

92. Ischaemum involutum G.Forst., F1. Ins. Austr. 73 (1786). - TYPE: Society Islands, J.R. Forster s.n. (lectotype UPS-THUNB [Herb. Thunberg 23948] [V-143195], designated by Nicolson \& Fosberg, Regnum Veg. 139: 247 (2004)).

= Thuarea involuta (G.Forst.) R.Br. ex Sm. in Rees, Cycl. 35(II, 70) [unpaged] (1817).

93. Ischaemum laeve Ridl., J. Straits Branch Roy. Asiat. Soc. 44: 207 (1905). - TYPE: Singapore, Galang, H.N. Ridley 9143 (lectotype SING [SING0054936], designated here; isolectotypes $\mathrm{G}, \mathrm{K})$.

= Ischaemum barbatum Retz., Observ. Bot. 6: 35 (1791).

94. Ischaemum macrurum Stapf ex Ridl., Fl. Malay Penins. 5: 203 (1925). - TYPE: Singapore, 27 October 1893, R.W. Hullett s.n. (lectotype K [K000290111], designated by Jansen, Reinwardtia 2: 298 (1953)).

= Ischaemum timorense Kunth, Révis. Gramin. 369, t. 98 (1830).

Notes. Later, Roberty (1960, p. 334) and Traiperm et al. (2012, p. 78) designated Ridley 10013 as lectotype, which is to be rejected. 
95. Ischaemum maculatum Stapf ex Ridl., Fl. Malay Penins. 5: 202 (1925). - TYPE: Peninsular Malaysia, Johore, road to Castlewood, 1898, H.N. Ridley 9170 (lectotype K [K000290178], first step designated by Roberty, Boissiera 9: 334 (1960), second step designated here; isolectotypes G, SING).

= Ischaemum feildingianum Rendle, J. Bot. 32: 101 (1894).

96. Ischaemum magnum Rendle, J. Bot. 32: 102 (1894). - TYPE: Singapore, Blakan Mati [Pulau Sentosa], October 1892, J.B. Feilding s.n. (lectotype BM [BM000959779], designated by Traiperm et al., Kew Bull. 67: 78 (2012); isolectotypes E, G, K).

= Ischaemum barbatum Retz., Observ. Bot. 6: 35 (1791).

97. Ischaemum muticum L., Sp. P1. 2: 1049 (1753). - TYPE: Anon. s.n. (lectotype LINN [Herb. Linn. 1214.1], designated by Renvoize in Jarvis et al., List Linn. Gen. Names Types 57 (1993)).

98. Ischaemum repens Roxb., Fl. Ind. 1: 325 (1820). - TYPE: [unpublished illustration] Icones Roxburghianae 2108 (lectotype K, designated here).

= Ischaemum muticum L., Sp. P1. 1049 (1753).

Notes. There seems to be no original material in the form of herbarium specimens available for this name (Forman, 1997) so the Roxburgh drawing in the Kew collection (on same sheet as 2109) is here selected as lectotype (Fig. 3).

99. Ischaemum rugosum Salisb., Icon. Stirp. Rar. 1: 1, t. 1 (1791). - TYPE: Locality unknown, J.G. König s.n. (lectotype BM [BM000959774], designated by Rendle, J. Bot. 32: 103 (1894)).

100. Ischaemum secundatum Walter, Fl. Carol. 249 (1788). - TYPE: USA, South Carolina, Beaufort County, Beaufort, roadbank on U.S. Rt. 21, 27 June 1956, H.E. Ahles 15602 (neotype GH [GH00277001], designated by Ward, J. Bot. Res. Inst. Texas 2(1): 479 (2008); isoneotypes GA, NCU, NY).

= Stenotaphrum secundatum (Walter) Kuntze, Revis. Gen. P1. 2: 794 (1891).

101. Ischaemum timorense Kunth, Révis. Gramin. 369, t. 98 (1830). - TYPE: Timor, Desfontaines s.n. (not traced). 
102. Lagurus cylindricus L., Syst. Nat., ed. 2, 2: 878 (1759). - TYPE: Gérard s.n. (lectotype LINN [Herb. Linn. 96.2], designated by Clayton \& Renvoize, Fl. Trop. E. Africa, Gram. (Part 3): 700. (1982)).

=Imperata cylindrica (L.) Raeusch., Nomencl. Bot. 3: 10 (1797).

103. Leersia hexandra Sw., Prodr. 21 (1788). - TYPE: O. Swartz s.n. (lectotype BM, designated by Clayton, Fl. Trop. E. Africa, Gram. (Part 1): 25 (1970); isolectotypes S, SBT).

104. Lophatherum gracile Brongn. in Duperr., Voy. Monde, Phan. 50, t. 8 (1831). - TYPE: Amboyna [Ambon], J.S.C. Dumont d'Urville s.n. (lectotype P [P00624191], designated here; possible isolectotype $\mathrm{P}[\mathrm{P} 00624190])$.

105. Melica latifolia Roxb. ex Hornem., Hort. Bot. Hafn. Suppl. 117 (1819). - TYPE: India, Colles Garrow [Garrow Hills], J.O. Voigt s.n. (neotype C [C10017246], designated here; possible isoneotype C [C10017247]).

= Thysanolaena latifolia (Roxb. ex Hornem.) Honda, J. Fac. Sci. Univ. Tokyo, Sect. 3, Bot. 3: 312 (1930).

Notes. The specimen in the Copenhagen herbarium that is annotated as holotype of Hornemann's name has 'mis. Voigt' written on the back - likely to mean 'misit Voigt', i.e. Voigt he sent. Voigt probably refers to Johann Otto Voigt who only took up a post at Serampore in 1826 (Stafleu \& Cowan, 1986). There is nothing on the specimen to indicate that Roxburgh saw it. It seems unlikely that the specimen represents original material, but in order to maintain continuity and as the specimen is reasonable, it is here selected as a neotype in the absence of any original material.

106. Milium cimicinum L., Mant. Pl. Altera 184 (1771). - TYPE: Anon. s.n. (neotype LINN [Herb. Linn. 83.2], designated by Clayton \& Renvoize, Fl. Trop. E. Africa, Gram. (Part 3): 617 (1982)).

= Alloteropsis cimicina (L.) Stapf, Fl. Trop. Afr. 9: 487 (1919).

107. Milium compressum Sw., Prodr. 24 (1788). - TYPE: Jamaica, R. Shakespear s.n. (lectotype BM [BM000578790], designated by Pohl \& Davidse, Fl. Mesoamer. 6: 357 (1991)).

=Axonopus compressus (Sw.) P. Beauv., Ess. Agrostogr. 12, 154, 167 (1812). 


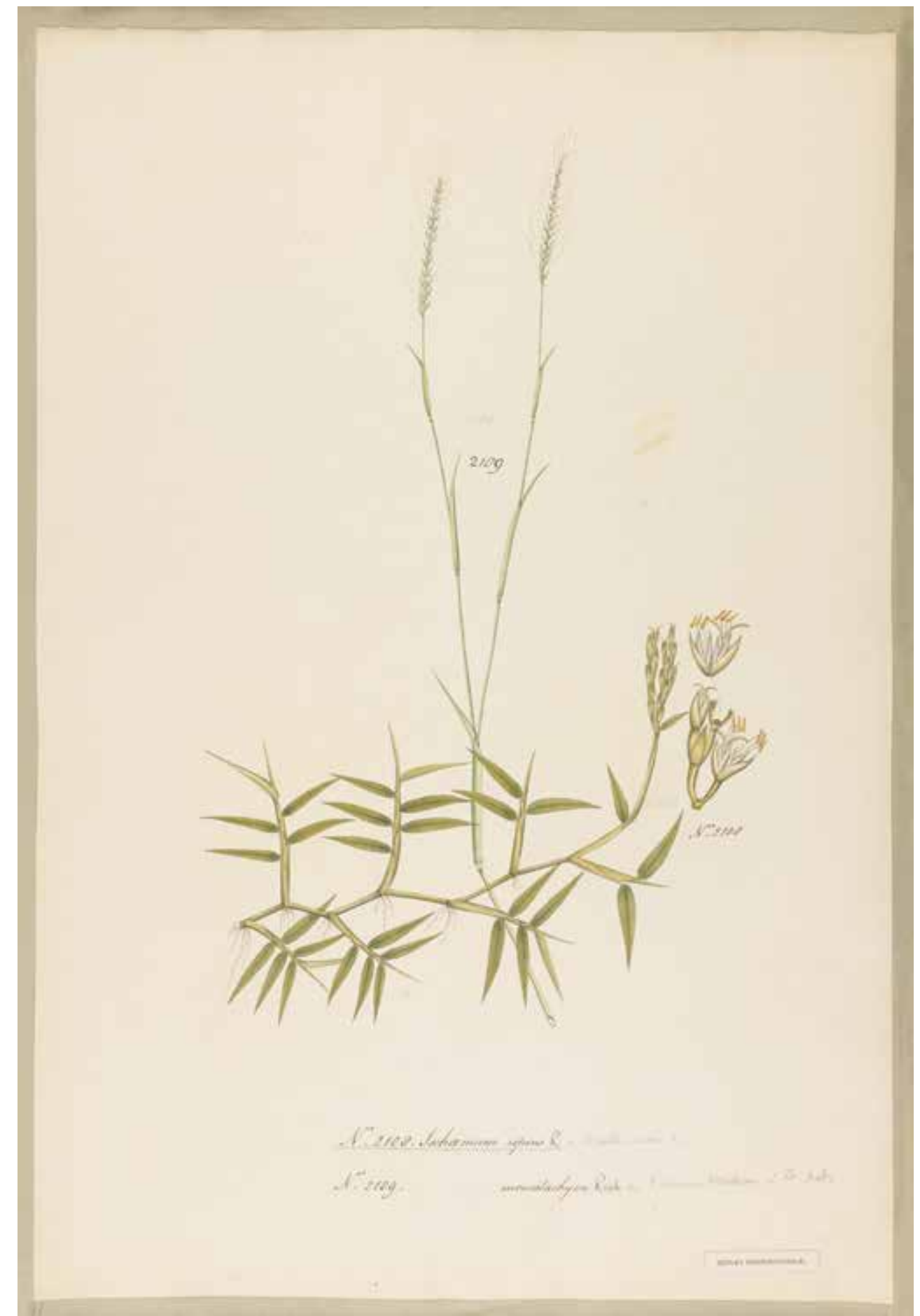

Fig. 3. Photograph of the lectotype of Ischaemum repens Roxb., Icones Roxburghianae 2108 in the Kew collection. Note that 2109 is drawn on the same sheet, but is excluded from the lectotype. Reproduced with the kind permission of the Director and the Board of Trustees, Royal Botanic Gardens, Kew. 
108. Milium globosum Thunb. in Murray, Syst. Veg., ed. 14: 109 (1784). - TYPE: Japan, C.P. Thunberg s.n. (lectotype UPS-THUNB [V-002041], designated here).

= Isachne globosa (Thunb.) Kuntze, Revis. Gen. P1. 2: 778 (1891).

109. Milium ramosum Retz., Observ. Bot. 6 (1791) 22. - TYPE: Anon. s.n. (lectotype LD [LD1222805], designated by Clayton \& Renvoize, Fl. Trop. E. Africa, Gram. (Part 3): 571 (1983)).

= Eriochloa procera (Retz.) C.E.Hubb., Bull. Misc. Inform. Kew 1930: 256 (1930).

110. Panicum L. sect. Gibbosae Hook.f., Fl. Brit. India 7: 28, 56 (1896). - TYPE: Panicum patens L. [= Cyrtococcum patens (L.) A.Camus] (lectotype designated here by Veldkamp).

= Cyrtococcum Stapf, Fl. Trop. Afr. 9:15 (1917)

111. Panicum accrescens Trin., Sp. Gram. 1: 164, t. 88 (1827). - TYPE: [published illustration] Trinius, Sp. Gram. 1: t. 88 (1827) (lectotype designated here by Veldkamp). = Cyrtococcum accrescens (Trin.) Stapf, Hooker's Icon. P1. 31: t. 3096 (1922).

Notes. Trinius cited "specimen nepalense" in the protologue but no candidate in the Trinius grass herbarium is available, so Trinius's plate is here selected as lectotype.

112. Panicum acutiglumum Steud., Syn. P1. Glumac. 1: 66 (1853). - TYPE: Peninsular Malaysia, Malacca, H. Cuming 2287 (lectotype K [K000290317], designated by Gilliland, Gard. Bull. Singapore 20: 314 (1964); isolectotypes A, E, GH, L, MO, P, W[3 sheets]).

= Hymenachne amplexicaulis (Rudge) Nees in Mart., F1. Bras. Enum. P1. 2: 276 (1829).

Notes. It seems likely that Cuming's collection was from Malacca rather than the Philippines.

113. Panicum amplexicaule Rudge, P1. Guian. 1: 21, t. 27 (1805). - TYPE: Guiana [French Guiana], J. Martin s.n. (holotype BM [BM000938736]).

= Hymenachne amplexicaulis (Rudge) Nees in Mart., F1. Bras. Enum. P1. 2: 267 (1829).

Notes. Stearn \& Williams (1957) provide an account of Rudge and his purchase of Martin's French Guiana collections as the spoils of war. 
114. Panicum angustum Trin., Sp. Gram. 3: t. 334 (1835). - TYPE: N. Wallich s.n. (lectotype LE [Herb. Trinius 578.1], designated here by Veldkamp).

= Sacciolepis indica (L.) Chase, Proc. Biol. Soc. Washington 21: 8 (1908).

115. Panicum arnottianum Nees ex Steud., Syn. P1. Glumac. 1: 59 (1853). - TYPE: Ceylon [Sri Lanka], 1829, J. Macrae s.n. [R. Wight 36] (lectotype B [B100366169], designated here; probable isolectotype CGE).

= Ottochloa nodosa (Kunth) Dandy, J. Bot. 69: 55 (1931).

Notes. The protologue refers to 'P. antidotale var. Hb Wight Ceylon, Java'. The sheet in B has a Herb. Wight propr. label that is annotated as 'Panicum antidotale var.' Other notes on the sheet seem to indicate that this specimen was in Nees von Esenbeck's herbarium having been received from John Lindley. There can be little doubt that this sheet represents original material for this name and it is here selected as lectotype. A sheet from Lindley's herbarium in CGE is also annotated with the name by Nees and is a Macrae collection from Ceylon dated 1829, but does not have any indication of a connection to Robert Wight. It is here considered a probable isolectotype.

116. Panicum auritum J.Presl ex Nees in Mart., F1. Bras. Enum. P1. 2: 176 (1829). - TYPE: Philippines, T.P.X. Haenke s.n. (lectotype MO [MO-156243], designated by Lamson-Scribner, Rep. (Annual) Missouri Bot. Gard. 10: 36, 46 (1899); isolectotypes BM, HAL, LE [Herb. Trinius 592.2], PR [sheet nos. 194822A and B, 194823A and B, 194824A, B and C], W[3 sheets]).

117. Panicum barbatum Lam., Tabl. Encycl. 1: 171 (1791). - TYPE: P. Commerson s.n. (lectotype P-LA [P00307246], designated by Veldkamp, Blumea 39: 375 (1994); isolectotype US (fragment)).

= Setaria barbata (Lam.) Kunth, Révis. Gramin. 1: 47 (1829).

118. Panicum brevifolium L., Sp. P1. 1: 59 (1753). - TYPE: India, Anon. s.n. (lectotype LINN [Herb. Linn. 80.64], designated by Clayton \& Renvoize, Fl. Trop. E. Africa, Gram. (Part 3): 496 (1982)).

119. Panicum burmanni Retz., Observ. Bot. 3: 10 (1783). - TYPE: J.G. König s.n. (lectotype LD [LD1244466], designated by Fischer, Bull. Misc. Inform. Kew 1932: 73 (1932); possible isolectotypes BM[3 sheets], C, LINN, S).

= Oplismenus burmanni (Retz.) P. Beauv., Ess. Agrostogr. 54, 168, 169, 170 (1812). 
Notes. The epithet burmanni, celebrating Burman as Burmannius, is correct and not to be corrected to burmannii or burmanii.

120. Panicum cambogiense Balansa, J. Bot. (Morot) 4: 142 (1890). - TYPE: Cambodge [Cambodia], 1875, A. Godefroy 62 (lectotype L [L0044815], designated by Veldkamp, Blumea 41: 195 (1996); isolectotypes K [K000290303] (fragment), P).

= Panicum luzonense J.Presl in C.Presl, Reliq. Haenk. 1: 308 (1830).

121. Panicum carinatum J.Presl in C.Presl, Reliq. Haenk. 1: 309 (1830). - TYPE: Philippines, Luzon, T.P.X. Haenke s.n. (lectotype MO [MO-2151007], designated by Lamson-Scribner, Rep. (Annual) Missouri Bot. Gard. 10: 36, 46 (1899); isolectotypes BM, HAL, LE [Herb. Trinius 620.1], PR [sheet nos. 194825A-E, 194826A-C], S (fragment), W[3 sheets]).

= Cyrtococcum accrescens (Trin.) Stapf, Hooker's Icon. P1. 31: t. 3096 (1922).

122. Panicum ciliare Retz., Observ. Bot. 4: 16 (1786). - TYPE: China, Cant[on], [H.P. Wennerberg s.n.] (lectotype LD [LD1244766], designated by Blake, Proc. Roy. Soc. Queensland 81: 10 (1970)).

= Digitaria ciliaris (Retz.) Koeler, Descr. Gram. 27 (1802).

123. Panicum colonum L., Syst. Nat., ed. 10, 2: 870 (1759). - TYPE: P. Browne s.n. (lectotype LINN [Herb. Linn. 80.23], designated by Hitchcock, Contr. U.S. Natl. Herb. 12: 119 (1908)).

= Echinochloa colona (L.) Link, Hort. Berol. 2: 209 (1833), as 'colonum'.

124. Panicum compositum L., Sp. Pl. 1: 57 (1753). - TYPE: Herb. Hermann vol. 3, fol. 45 (lectotype BM [BM000621970], designated by Hitchcock, U.S.D.A. Bull. 772: 238 (1920)).

= Oplismenus compositus (L.) P.Beauv., Ess. Agrostogr. 54, 168, 169 (1812).

125. Panicum concinnum Nees in Hooker's J. Bot. Kew Gard. Misc. 2: 97 (1850), nom. illeg., non Schrad. (1838). - TYPE: H. Cuming 2284 (lectotype CGE [06335(CGE)], designated by Veldkamp, Blumea 41: 203 (1996); isolectotypes E, K, L, MO, P).

= Panicum sarmentosum Roxb., F1. Ind. 1: 311 (1820). 
126. Panicum crassiapiculatum Merr., Philipp. J. Sci. 1, Suppl. 5: 356 (1906). - TYPE: Philippines, Balabac Island, October 1906, E.D. Merrill 5387 (lectotype US [US00604218], designated here by Veldkamp; isolectotype W).

= Acroceras munroanum (Balansa) Henrard, Blumea 3: 445 (1940).

127. Panicum crus-galli L., Sp. P1. 1: 56 (1753), as 'Crusgalli'. - TYPE: [North America] Anon. s.n. [P. Kalm leg.] (lectotype LINN [Herb. Linn. 80.18], designated by Hitchcock, Contr. U.S. Natl. Herb. 12: 117 (1908)).

=Echinochloa crus-galli (L.) P.Beauv., Ess. Agrostogr. 53, 161, 169, t. 11, f. 2 (1812).

128. Panicum dactylon L., Sp. P1. 1: 58 (1753). - TYPE: Sweden, Uppsala, cultivated, Anon. s.n. (lectotype LINN [Herb. Linn. 80.35], designated by Clayton \& Harlan, Kew Bull. 24: 186 (1970)).

= Cynodon dactylon (L.) Pers., Syn. P1. 1: 85 (1805).

129. Panicum dilatatum Steud., Syn. P1. Glumac. 1: 39 (1853). - TYPE: India, Courtallam, R. Wight 2340 (lectotype K [K000245353], first step designated by Henrard, Monogr. Gen. Digitaria 241 (1950), second step designated here).

= Digitaria setigera Roth, Syst. Veg. 2: 474 (1817).

130. Panicum glumare Trin., Gram. Panic. 143 (1826). - TYPE: New Zealand (probably wrongly localised), Anon. s.n. (lectotype LE [Herb. Trinius 727.1], designated by Veldkamp, Blumea 41: 420 (1996)).

= Urochloa glumaris (Trin.) Veldkamp, Blumea 41: 420 (1996).

131. Panicum hirtifolium Ridl., Mat. Fl. Malay. Penins. 3: 141 (1907). - TYPE: Peninsular Malaysia, Selangor, Semangkok, 1904, H.N. Ridley 12046 (lectotype SING [SING0054931], designated here by Veldkamp; isolectotype K [K000290294]).

= Panicum brevifolium L., Sp. P1. 1: 59 (1753).

132. Panicum incomtum Trin., Gram. Panic. 200 (1826). - TYPE: Philippines, Manila, A. von Chamisso s.n. (lectotype LE [Herb. Trinius 760.2], designated here by Veldkamp).

= Panicum sarmentosum Roxb., Fl. Ind. 1: 311 (1820). 
133. Panicum italicum L., Sp. P1. 1: 56 (1753). - TYPE: Anon s.n. (lectotype L (Herb. van Royen) [sheet 912.356-242], designated by Veldkamp in Cafferty et al., Taxon 49: 253 (2000)).

= Setaria italica (L.) P.Beauv., Ess. Agrostogr. 51, 170, 178 (1812).

134. Panicum latifolium L. var. majus Hook.f., Fl. Brit. India 7: 39 (1896). - TYPE: Peninsular Malaysia, Perak. Goping, October 1888, King's Collector [H.H. Kunstler] 876 (lectotype K [K000290228], designated here or possibly holotype; isolectotypes CAL, SING).

= Acroceras tonkinense (Balansa) C.E.Hubb. ex Bor, Indian Forest Rec. 1: 78 (1938).

135. Panicum laxum Sw., Prodr. 23 (1788). - TYPE: Jamaica, O.P. Swartz s.n. (lectotype S [S-R-3977], designated by Hitchcock, Contr. U.S. Natl. Herb. 12: 143 (1908); possible isolectotypes UPS [2 sheets], US (fragment)).

136. Panicum luzonense J.Presl in C.Presl, Reliq. Haenk. 1: 308 (1830). - TYPE: Philippines, Luzon, Sorsogon, T.P.X. Haenke s.n. (lectotype PR [sheet no. 612343], designated by Veldkamp, Blumea 41: 195 (1996); isolectotype LE [Herb. Trinius 800.1], W).

137. Panicum malaccense Trin., Gram. Panic. 204 (1826). - TYPE: J.G. Klein 715 (lectotype LE [Herb. Trinius 806.1] (fragment), designated by Prakash \& Jain, Fasc. Fl. India 14: 39 (1984); isolectotypes B-W [B-W01737010], HAL (fragment)).

= Sphaerocaryum malaccense (Trin.) Pilg., Feddes Repert. 45: 2 (1938).

138. Panicum maximum Jacq., Icon. P1. Rar. 1: 2, t. 13 (1781). - TYPE: Guadeloupe, Anon. s.n. (lectotype W [W·0011326], designated by Zuloaga, Darwiniana 22: 24 (1979); isolectotype BM).

= Urochloa maxima (Jacq.) R.D.Webster, Austral. Paniceae 241 (1987).

139. Panicum meyerianum Nees, Fl. Afr. Austral. Ill. 32 (1841). - TYPE: CBSP [Capitis Bonae Spei Promontorium = Cape of Good Hope], J.F. Drège s.n. (lectotype LE [Herb. Trinius 825.2], designated here by Veldkamp; possible isolectotypes G[3 sheets], HAL, K, P, S, TUB, W).

=Eriochloa meyeriana (Nees) Pilg. in Engler, Nat. Pflanzenfam., ed. 2, 14e: 56 (1940). 
140. Panicum microbachne J.Presl in C.Presl, Reliq. Haenk. 1: 298 (1830). - TYPE: Philippines, Luzon, Sorsogon, T.P.X. Haenke s.n. (lectotype PR [sheet no. 24357], designated by Reeder, J. Arnold Arbor. 29: 292 (1948), excluding central plant labelled Mexico; isolectotypes BISH, HAL, US (fragment), W).

= Digitaria setigera Roth, Syst. Veg. 2: 474 (1817).

Notes. When Reeder designated the lectotype for Panicum microbachne he excluded the central plant. It is not clear that this plant is currently labelled as being from Mexico. The specimen was remounted in recent decades (O. Sída pers. comm.) and this may have led to a different arrangement of plants and labels. The collecting locations of Haenke's specimens are frequently questionable.

141. Panicum miliiforme J.Presl, Reliq. Haenk. 1: 300 (1830). - TYPE: Philippines, Luzon, T.P.X. Haenke s.n. (lectotype MO [MO-157631], designated by LamsonScribner, Rep. (Annual) Missouri Bot. Gard. 10: 36, 47 (1899); isolectotypes HAL, PR [sheet nos. 194810A and B], US (fragment), W[3 sheets]).

= Urochloa subquadripara (Trin.) R.D.Webster, Austral. Paniceae 252 (1987).

142. Panicum minutulum Gaudich. in Freyc., Voy. Uranie: 410 (1829). - TYPE: Mariana Islands, C. Gaudichaud-Beaupré s.n. (lectotype P [P01934348], first step designated by Fosberg \& Sachet, Micronesica 18: 55-56 (1984), second step designated here by Veldkamp; possible isolectotypes B, P [P01934347]).

= Isachne minutula (Gaudich.) Kunth, Rév. Gram. 2: 407, t. 117 (1831).

143. Panicum multinode J.Presl in C.Presl, Reliq. Haenk. 1: 303 (1830), nom. illeg., non Lam. (1798). = Panicum nodosum Kunth, Enum. Pl. 1: 97 (1833). - TYPE: [Philippines, Luzon, Sorsogon] T.P.X. Haenke s.n. (lectotype MO [MO-2114722], designated by Lamson-Scribner, Rep. (Annual) Missouri Bot. Gard. 10: 36, 47 (1899); isolectotypes B, BM, HAL, LE [Herb. Trinius 844.1], PR [sheet nos. 194817A and B, 194818A and B], US (fragment), W[3 sheets]).

= Ottochloa nodosa (Kunth) Dandy, J. Bot. 69: 55 (1931).

144. Panicum munroanum Balansa, J. Bot. (Morot) 4: 140 (1890). - TYPE: Sri Lanka, CP 3244 (lectotype K [K000245280], right-hand plant, designated here).

=Acroceras munroanum (Balansa) Henrard, Blumea 3: 445 (1940).

145. Panicum muticum Forssk., Fl. Aegypt.-Arab. 20 (1775). - TYPE: Egypt, Rosetta, P. Forsskål 86 (lectotype C [C10002726], designated by Blake, Proc. Roy. Soc. Queensland 81: 5 (1969); isolectotype BM).

= Urochloa mutica (Forssk.) T.Q.Nguyen, Novosti Sist. Vyssh. Rast. 3: 13 (1966). 
146. Panicum myosuroides R.Br., Prodr. 1: 189 (1810). - TYPE: New Holland, [Australia], Endeavour River, 1770, J. Banks \& D. Solander s.n. (lectotype BM [BM000793678], first step designated by Webster, Pan. Austral. 202 (1987), second step designated here; possible isolectotypes BM [BM000793679], CANB [2 sheets]). = Sacciolepis myosuroides (R.Br.) Chase ex E.G.Camus \& A.Camus, F1. Indo-Chine 7: 460 (1922).

Notes. For the entry under this name in Chase \& Niles (1962, p. 551), "T" = Littora novae hollandiae intra tropicum.' is not a direct reference to the type but one of Robert Brown's codes for general localities in New Holland [Australia].

147. Panicum ovalifolium Poir., Encycl., Suppl. 4: 279 (1816). - TYPE: Guinea, Anon. s.n. (holotype P [P00442141]).

= Panicum brevifolium L., Sp. P1. 1: 59 (1753).

148. Panicum pallidefuscum Schumach., Beskr. Guin. Pl. 58 (1827). - TYPE: Guinea [Ghana], P. Thonning 344 (lectotype C [C10004269], designated here; isolectotypes C[4 sheets], K (fragment)).

= Setaria parviflora (Poir.) Kerguélen, Lejeunia n.s. 120: 161 (1987).

149. Panicum palmifolium J.Koenig, Naturforscher (Halle) 23: 208 (1788), as 'palmaefolium'. - TYPE: Anon. s.n. (neotype K [K000245334], designated here).

= Setaria palmifolia (J.Koenig) Stapf, J. Linn. Soc., Bot. 42: 186 (1914).

Notes. Veldkamp (1994, p. 379) referred to the holotype of Panicum palmifolium as a Koenig collection in BM. No such specimen has been located. Neither is there a relevant Koenig specimen in the Desfontaines Herbarium in Florence (C. Nepi, pers. comm.), contrary to what is stated in Tropicos [www.tropicos.org]. We here select as neotype a specimen in K which came from J.P. Rottler's herbarium. This specimen bears a ticket in Rottler's hand inscribed with Koenig's name and place of publication. It was annotated by Veldkamp in 2010 as holotype of Panicum palmifolium. Conceivably the specimen could have come from or been identified by Koenig, but given the high degree of doubt over this we prefer to designate it as a neotype.

150. Panicum paludosum Roxb., Fl. Ind. 1: 310 (1820). - TYPE: W. Roxburgh s.n. [EIC 8711[A]] (lectotype K-W [K001131333], designated here).

Notes. There are Roxburgh specimens of this species in BM, BR and K. Only the specimen in the Wallich Herbarium bears Roxburgh's handwritten ticket with the name, so it is selected as the lectotype. 
151. Panicum patens L., Sp. P1. 1: 58 (1753). - TYPE: Anon. s.n. (lectotype LINN [Herb. Linn. 80.63], designated by Mitra \& Jain in Manilal, Bot. Hist. Hort. Malabaricus 151 (1980)).

=Cyrtococcum patens (L.) A.Camus, Bull. Mus. Natl. Hist. Nat. 27: 118 (1921).

152. Panicum pictum J.Koenig, Naturforscher (Halle) 23: 204 (1788). - TYPE: J.G. König s.n. (lectotype LD [1220586], designated here).

=Echinochloa picta (J.Koenig) P.W.Michael, Philipp. J. Weed Sci. 5: 18 (1978).

153. Panicum piligerum F.Muell. ex Benth., Fl. Austral. 7: 477 (1878). - TYPE: Australia, Victoria River, F. von Mueller s.n. (lectotype K [K000958892], first step designated by Hughes, Bull. Misc. Inform. Kew 1923: 315 (1923), second step designated by Veldkamp, Blumea 41: 425 (1996), or possibly holotype; isolectotypes BRI (fragment), MEL).

= Urochloa piligera (F.Muell. ex Benth.) R.D.Webster, Austral. Paniceae 246 (1987).

154. Panicum polystachion L., Syst. Nat., ed. 10, 2: 870 (1759). - TYPE: [published illustration] Rumphius, Herb. Amb. 6: t. 7, f. 2B (1750) (lectotype designated by Merrill, Interpret. Rumph. Herb. Amb. 91 (1917)).

= Setaria flava (Nees) Kunth, Rév. Gram. 1: 46 (1829).

Notes. Panicum polystachion L. was long used for a species of Panicum that was recently transferred to Cenchrus and was lectotypified in this sense by van der Zon (1992). However, Merrill had already lectotypified the name to the Rumphius plate cited by Linnaeus which represents Setaria flava (Nees) Kunth. The correct name for Panicum polystachion auctt. plur. becomes Cenchrus setosus Sw.

155. Panicum pruriens Fisch. ex Trin., Gram. Panic. 77 (1826). - TYPE: [French Polynesia, Marquesas], Nukahiva, G.H. von Langsdorff s.n. (lectotype LE [Herb. Trinius 906.5], designated by Henrard, Monogr. Gen. Digitaria 598 (1950); isolectotype BM).

= Digitaria setigera Roth, Syst. Veg. 2: 474 (1817).

156. Panicum radicosum J.Presl in C.Presl, Reliq. Haenk. 1: 297 (1830). - TYPE: Philippines, Luzon, Sorsogon, T.P.X. Haenke s.n. (lectotype PR [sheet no. 194788], designated by Blake, Proc. Roy. Soc. Queensland 81: 19 (1969)).

= Digitaria radicosa (J.Presl) Miq., Fl. Ned. Ind. 3: 437 (1857). 
157. Panicum repens L., Sp. P1., ed. 2, 1: 87 (1762). - TYPE: C. Alströmer $2 a$ (lectotype LINN [Herb. Linn. 80.47], designated by Hitchcock \& Chase, Contr. US Natl. Herb. 15: 85 (1910)).

158. Panicum reticulatum Thwaites ex Trimen, J. Bot. 23: 271 (1885), nom. illeg., non Torrey (1852). = Panicum caesium Nees ex Hook. f., Fl. Brit. India 7: 48 (1896), nom. illeg., non Nees (1837). = Panicum cruciabile Chase, J. Arnold Arbor. 20: 309 (1939). - TYPE: Sri Lanka, CP 3890 (lectotype PDA, designated by Veldkamp, Blumea 41: 195 (1996)).

= Panicum luzonense J.Presl, Reliq. Haenk. 1: 308 (1830).

159. Panicum ridleyi Hack., Bot. Tidsskr. 24: 98 (1901). - TYPE: Peninsular Malaysia, Malacca, Pulau Besar, September 1889, H.N. Ridley 61 (lectotype W [1916-0024255], designated here; isolectotype SING).

= Acroceras tonkinense (Balansa) C.E.Hubb. ex Bor, Indian Forest Rec. 1: 78 (1938).

160. Panicum rubiginosum Steud., Syn. Pl. Glumac. 1: 50 (1853). - TYPE: Philippines, H. Cuming 551 (lectotype P [P00642103], designated here; isolectotypes BM, CGE, G [3 sheets], K, L, MO, P [2 sheets], W).

= Setaria parviflora (Poir.) Kerguélen, Lejeunia, n.s., 120: 161 (1987).

161. Panicum sarmentosum Roxb., F1. Ind. 1: 311 (1820). - TYPE: W. Roxburgh s.n. (lectotype BR [BR0000006883584], designated here).

Notes. The Roxburgh specimen in BR seems to be the only original herbarium material available for this name. It bears a label in Roxburgh's hand.

162. Panicum sphacelatum Schumach., Beskr. Guin. P1. 58 (1827). - TYPE: Guinea (Ghana), P. Thonning s.n. (lectotype C [C10004281], designated by Hepper, W. Afr. Herb. Isert Thonning 150 (1976); possible isolectotypes C [[C10004288], K).

= Setaria sphacelata (Schumach.) Stapf \& C.E.Hubb., Fl. Trop. Afr. 9: 795 (1930).

163. Panicum subquadriparum Trin., Gram. Panic. 145 (1826). - TYPE: Marianas, insula Guahan, J.F. von Eschscholtz \& A. de Chamisso s.n. (lectotype LE [Herb. Trinius 974.1], left-hand specimen, designated by Veldkamp, Blumea 41: 429 (1996)). = Urochloa subquadripara (Trin.) R.D.Webster, Austral. Paniceae 252 (1987). 
164. Panicum tenuissimum Mart. ex Schrank, Denkschr. Bayer. Bot. Ges. Regensb. 2: 26 (1822). - TYPE: Brazil, in sylvis iter Sebastianopolin et Soteropolin, C.F.P. von Martius 5495 (lectotype M [M-0104367], first step designated by Renvoize, Grasses Bahia 61 (1984), second step designated here).

= Sporobolus tenuissimus (Mart. ex Schrank) Kuntze, Revis. Gen. P1. 3: 369 (1898).

Notes. There are two Martius specimens of this taxon in the Munich Herbarium. One has what seems to be an original field ticket numbered 5495 and the other one labelled 5496. The former, which seems to be treated as the 'holotype', is here selected as lectotype.

165. Panicum tonkinense Balansa, J. Bot. (Morot) 4: 140 (1890). - TYPE: Vietnam, Bois des environs de Tu-Phap, May 1887, B. Balansa 1646 (lectotype L [L0043504], designated here by Veldkamp; isolectotypes L[2 sheets], K, P, US).

= Acroceras tonkinense (Balansa) C.E.Hubb. ex Bor, Indian Forest Rec. 1: 78 (1938).

166. Panicum trichocladum Hack. ex K.Schum., Pflanzenw. Ost-Afrikas 2/3C: 102 (1895). - TYPE: Tanzania, Kilimandscharo, July 1887, H. Meyer 140 (lectotype B [B 10 0715462], designated by Vorontsova, Candollea 73: 178 (2018); isolectotype US [US00140067]).

167. Paspalum bicorne Lam., Tabl. Encycl. 1: 176 (1791). - TYPE: P. Sonnerat s.n. (holotype P-LA [P00563991]; isotype US (fragment)).

= Digitaria bicornis (Lam.) Roem. \& Schult., Syst. Veg. 2: 470 (1817).

168. Paspalum cartilagineum J.Presl in C.Presl, Reliq. Haenk. 1: 216 (1830). - TYPE: [Philippines, Luzon, Sorsogon] T.P.X. Haenke s.n. (lectotype MO [MO-2114871], designated by Lamson-Scribner, Rep. (Annual) Missouri Bot. Gard. 10: 36, 49 (1899); isolectotypes HAL, K (fragment), LE [Herb. Trinius 0529.04-b], US (fragment), W). = Paspalum scrobiculatum L. var. bispicatum Hack. ex Merr., Fl. Manila 86 (1912).

169. Paspalum chinense Nees in Hook. \& Arn., Bot. Beechey Voy. (5): 231 (1836), as 'Paspalus chinensis'. - TYPE: China, Macao, Summer 1830, G.H. Vachell s.n. (lectotype CGE [06343(CGE)], designated by Henrard, Monogr. Gen. Digitaria 122 (1950)).

= Digitaria violascens Link, Hort. Berol. 1: 229 (1827).

Notes. Noltie (2010) showed that this part of the Hooker and Arnott work was published in 1836 rather than 1837 as widely stated. 
170. Paspalum commersonii Lam., Tabl. Encycl. 1: 175, t. 43 (1791). - TYPE: Isle de France [Mauritius], P. Commerson s.n. (lectotype P-JU [P00665168] [Herb. Juss. 2629], first step designated by Clayton, Kew Bull. 30: 101 (1975), second step designated here; isolectotypes L, P-LA [P00563992]).

= Paspalum scrobiculatum L. var. bispicatum Hack. ex Merr., Fl. Manila: 86 (1912).

Notes. The sheet in the Lamarck Herbarium bears two labels. On the lower left there is a field label with 'Isle de France C. ${ }^{\text {om' }}$, and on the right one with 'Coromandel C. ${ }^{\text {om' }}$. If these are separate gatherings from Mauritius and India, the juxtaposing of labels and herbarium material would indicate that the Mauritius specimen consists of a sterile tiller and a section of rhizome. It therefore seems prudent to select the better specimen from the de Jussieu herbarium as the second-step lectotype.

171. Paspalum conjugatum P.J.Bergius, Acta Helv. Phys.-Math. 7: 129, t. 8 (1772). - TYPE: Suriname, 4 August 1755, D. Rolander s.n. (lectotype SBT [Bergius Herb. 1.2.7.36], designated by de Koning \& Sosef, Blumea 30: 290 (1985)).

172. Paspalum fissifolium Raddi, Agrostogr. Bras. 26 (1823), as 'Paspalus fissifolius'. - TYPE: Raddi s.n. (lectotype PI, designated by Judziewicz, Fl. Guianas, ser. A, Phanerogams 8: 98 (1990); isolectotypes BAA, BM, FI, G, K, US (fragment), W).

= Axonopus fissifolius (Raddi) Kuhlm., Relat. Commiss. Linhas Telegr. Estratég. Matto Grosso Amazonas 5(11): 87 (1922).

Notes. Baldini \& Longhi Wagner (2006, p. 473) selected the PI sheet as lectotype but this had already been effectively done by Judziewicz (1990).

173. Paspalum fuscescens J.Presl in C.Presl, Reliq. Haenk. 1:213, 351 (1830). - TYPE: [USA, California, Monte-Rey] T.P.X. Haenke s.n. (lectotype MO [MO-440063], designated by Lamson-Scribner, Rep. (Annual) Missouri Bot. Gard. 10: 36, 49 (1899); isolectotypes BM, BR, HAL, L, PR, US (fragment), W).

= Digitaria fuscescens (J.Presl) Henrard, Meded. Rijks-Herb. 61: 8 (1930).

174. Paspalum longiflorum Retz., Observ. Bot. 4: 15 (1786). - TYPE: J.G. König s.n. (lectotype LD, designated by Henrard, Monogr. Gen. Digitaria 410 (1950); isolectotypes $\mathrm{BM}, \mathrm{K})$.

= Digitaria longiflora (Retz.) Pers., Syn. P1. 1: 85 (1805). 
175. Paspalum longifolium Roxb., Fl. Ind. 1: 283 (1820). - TYPE: W. Roxburgh s.n. (lectotype BM [BM000959597], designated by de Koning \& Sosef, Blumea 30: 297 (1985)).

= Paspalum sumatrense Roth in Roem. \& Schult., Syst. Veg. 2: 316 (1817).

176. Paspalum molle J.Presl in C.Pres1, Reliq. Haenk. 1: 213 (1830), nom. illeg., non Poir. (1804). - Paspalum mollicomum Kunth, Enum. P1. 1: 47 (1833). - TYPE: [Philippines, Luzon] T.P.X. Haenke s.n. (lectotype MO [MO-2151004], designated by Lamson-Scribner, Rep. (Annual) Missouri Bot. Gard. 10: 36, 50 (1899); isolectotypes B, BM, LE [Herb. Trinius 495.1], PR [197877-B], US (fragment), W).

= Digitaria mollicoma (Kunth) Henrard, Blumea 1: 97 (1934).

177. Paspalum orbiculare G.Forst., Fl. Ins. Austr. 7 (1786). - TYPE: Forster s.n. (syntypes C [C10017146], K [K000215190], UPS).

Notes. Nicolson \& Fosberg (2004, p. 239) confirm that there is no original material for this name in the Goettingen Herbarium as widely cited.

178. Paspalum platicaulon Poir., Tabl. Encycl. 5: 34 (1804). - TYPE: Puerto Rico, Ledru s.n. (holotype P-LA [P00564007]).

= Axonopus compressus (Sw.) P.Beauv., Ess. Agrostogr. 12, 154, 167 (1812).

179. Paspalum plicatulum Michx., Fl. Bor.-Amer. 1: 45 (1803). - TYPE: USA, Hab in Georgiâ, Floridâ, A. Michaux 2 (lectotype P-MICH [P00670149], designated by Hitchcock, Contr. U.S. Natl. Herb. 12: 143, 144, 146 (1908); isolectotypes P-JUSS [Herb. Juss. 2636], US (fragment)).

180. Paspalum scrobiculatum L., Mant. Pl. Altera 29 (1767). - TYPE: Sweden, Uppsala, cultivated, Anon. s.n. (lectotype LINN [Herb. Linn. 79.4], designated by Clayton, Kew Bull. 30: 101 (1975)).

181. Paspalum scrobiculatum L. var. bispicatum Hack. ex Merr., F1. Manila 86 (1912). - TYPE: Philippines, Luzon, Province of Rizal, San Pedro Macati, July 1910, E.D. Merrill 272 (lectotype W [1916-0041752], designated here).

Notes. Merrill did not cite any specimens in the protologue but did give locality information. The type was referred to by de Koning \& Sosef (1985, p. 305) as Kneucker Exsiccata 803 with a holotype in W. This would be correctable to an effective lectotypification if it were original material but de Koning \& Sosef cited an 
incorrect Kneucker (1915) protologue and also noted they had not seen this sheet. We have not been able to locate it and it is possible this specimen may not have existed. Instead there is a specimen in W which was collected by Merrill and annotated by Hackel and has the locality data from Merrill's protologue. In the absence of evidence that there ever was a Kneucker Exsiccata 803 specimen in W, or that it is original material, the Merrill collection is selected here as lectotype.

182. Paspalum sumatrense Roth in Roem. \& Schult., Syst. Veg. 2: 316 (1817). - TYPE: Sumatra, B. Heyne s.n. (lectotype K (fragment) [K000290255], designated here).

Notes. Benjamin Heyne collected specimens in Sumatra in 1812 (van SteenisKruseman, 1950). He passed the collections, together with many from India, to Albrecht Roth who wrote them up. Unfortunately, Roth's herbarium was largely destroyed in Berlin during the Second World War (Bor, 1955). However, there is a sheet at Kew with a single spikelet taken from Roth's type in 1914 when it was in Oldenburg and a poor photograph. The specimen is here designated lectotype for Roth's name.

183. Paspalum vaginatum Sw., Prodr. 21 (1788). - TYPE: fl. ind. occ. ex ins. Sti Crucis Am., O.P. Swartz s.n. (lectotype S [S-R-4070], first step designated by Clayton \& Renvoize, Fl. Trop. E. Africa, Gram. (Part 3): 609 (1982), second step designated by de Koning \& Sosef, Blumea 30: 310 (1985); possible isolectotypes S, UPS).

184. Pennisetum purpureum Schumach., Beskr. Guin. Pl. 44 (1827). - TYPE: Guinea [Ghana], P. Thonning 355 (lectotype C [C10004308], designated by Hepper, W. Afr. Herb. Isert Thonning 149 (1976); isolectotype BM).

= Cenchrus purpureus (Schumach.) Morrone, Ann. Bot. (Oxford) 106: 129 (2010).

185. Perotis hordeiformis Nees in Hook. \& Arnott, Bot. Beechey Voy. (6): 247-248 (1838). - TYPE: N.W. India, J.F. Royle 280 (lectotype K [K000245204], designated by Veldkamp \& Steenbergen, Austrobaileya 3: 610 (1992); isolectotypes B, LE [Herb. Trinius 390.1], LIV).

=Perotis indica (L.) Kuntze, Rev. Gen. Pl. 2: 787 (1891).

186. Phalaris zizanioides L., Mant. Pl. Altera 2: 183 (1771). - TYPE: India, J.G. König s.n. (lectotype LINN [Herb. Linn. 78.12], designated by van der Zon, Wageningen Agr. Univ. Pap. 92-1: 409 (1992)).

= Chrysopogon zizanioides (L.) Roberty, Bull. Inst. Fondam. Afrique Noire, Sér. A, Sci. Nat. 22: 106 (1960). 
187. Pharus urceolatus Roxb., Fl. Ind. ed. 1832, 3: 611 (1832). - TYPE: $W$. Roxburghs.n.(lectotypeBM[BM000959309], designated by Soderstrometal., Smithson. Contr. Bot. 65: 3 (1987)).

= Scrotochloa urceolata (Roxb.) Judz., Phytologia 56: 300 (1984).

188. Phleum indicum Houtt., Nat. Hist. 2, 13: 198, t. 90, f. 2 (1782). - TYPE: Anon. s.n. (lectotype G-PREL [G00096222)], designated by Veldkamp in Wijnands et al., Candollea 72: 184 (2017)).

= Polytrias indica (Houtt.) Veldkamp, Blumea 36: 180 (1991).

189. Poa atrovirens Desf., Fl. Atlant. 1: 73, t. 14 (1798). - TYPE: Algeria, Desfontaines s.n. (lectotype LE (right-hand plant only), designated by Jain, J. Indian Bot. Soc. 50: 129 (1971); isolectotypes C, FI, P).

= Eragrostis atrovirens (Desf.) Trin. ex Steud., Nomencl. Bot., ed. 2, 1: 562 (1840).

190. Poa chinensis L., Sp. Pl. 1: 69 (1753). - TYPE: Osbeck s.n. (lectotype LINN [Herb. Linn. 87.32], designated by Phillips, Fl. Trop. E. Africa, Gram. (Part 2): 279 (1974)).

= Dinebra chinensis (L.) P.M.Peterson \& N.Snow, Ann. Bot. (Oxford) 109: 1326 (2012).

191. Poa amabilis L., Sp. P1. 1: 68 (1753). - TYPE: Hermann vol. 2, fol. 59, no. 46 (lectotype BM [BM000621703], designated by Veldkamp, Taxon 49: 254 (2000)).

= Eragrostis tenella (L.) P.Beauv. ex Roem. \& Schult., Syst. Veg. 2: 576 (1817).

192. Poa elegans Roxb., Fl. Ind. 1: 339 (1820), nom. illeg., non Poir. (1804), nec R.Br. (1810). - Poa chariis Schult., Mant. 2: 314 (1824). - TYPE: [unpublished illustration] Icones Roxburghianae 841 (lectotype K, designated here).

= Eragrostis atrovirens (Desf.) Trin. ex Steud., Nomencl. Bot., ed. 2, 1: 562 (1840).

Notes. No herbarium material directly associated with this name has been discovered. Therefore, Roxburgh's drawing is here selected as lectotype.

193. Poa gangetica Roxb., Fl. Ind. 1: 341 (1820). - TYPE: India orientalis, 1813, W. Roxburgh s.n. (lectotype BM [BM000578754], designated by Clayton, Fl. Trop. E. Africa, Gram. (Part 2): 217 (1974)).

= Eragrostis gangetica (Roxb.) Steud., Syn. P1. Glumac. 1: 266 (1854). 
194. Poa panicea Retz., Observ. Bot. 3: 11 (1783). - TYPE: P.J. Bladh s.n. (holotype LD [1296347]; isotype BRI (fragment)).

= Dinebra panicea (Retz.) P.M.Peterson \& N.Snow, Ann. Bot. (Oxford) 109: 1326 (2012).

195. Poa pilosa L., Sp. P1. 1: 68 (1753). - TYPE: [published illustration] 'Gramen paniculis elegantissimus, majus, locustis purpureo-spadiceis, minoribus', Scheuchzer, Agrostographia: t. 4, f. 3 (1719) (lectotype designated by Koch, Illinois Biol. Monogr. 48: 24 (1974). Italy, Piemonte, Alluvium der Sesia bei Vercelli und an den Wegen zwischen den Reisfeldern von Oldenico unweit Vercelli in Oberitalien, 9-10 August 1902, A. Kneucker Gram. exsicc. XII, No. 344 (epitype B, designated by Scholz in Cafferty et al., Taxon 49: 256 (2000); possible isoepitypes L, W).

= Eragrostis pilosa (L.) P.Beauv., Ess. Agrostogr. 71, 162, 175 (1812).

Notes. Koch (1974) cited the type illustration as 'Pl. 4 tab. 3; instead of 'tab. 4 fig. 3', but we treat the misuse of 'tabula' as a correctible error.

196. Poa polymorpha R.Br., Prodr. 1: 180 (1810), nom. illeg., non Wibel (1799). - Poa brownii Kunth, Révis. Gramin. 112 (1829), as 'brownei'. - TYPE: Australia, Shoalwater Bay, 3 September 1802, R. Brown 6284 (lectotype BM [BM000991612], designated by Lazarides, Austral. Syst. Bot. 10: 101 (1997); isolectotypes E [E00381774], K, US (fragment)).

= Eragrostis brownii (Kunth) Nees in Wight, Cat. Indian P1. 105 (1834).

197. Poa tenella L., Sp. P1. 1: 69 (1753). - TYPE: India, Anon. s.n. (lectotype LINN [Herb. Linn. 87.33], designated by Mitra \& Jain, Bot. Hist. Hort. Malabaricus 151 (1980)).

= Eragrostis tenella (L.) P.Beauv. ex Roem. \& Schult., Syst. Veg. 2: 576 (1817).

198. Poa tenuifolia A.Rich., Tent. Fl. Abyss. 2: 425 (1851). - TYPE: Ethiopia, locis incultis vallium prope Adoam, 18 September 1837, G.W.H. Schimper, Iter Abyssinicum. Sectio prima: plantae Adoënses 92 (lectotype P [P00439420], first step designated by Phillips, F1. Ethiopia 7: 122 (1995), second step designated here; isolectotypes B, BR, FR, G[4 sheets], GOET, K, L, MPU, P[2 sheets] [P00439421, P00439422], TUB[2 sheets], US (fragment), W[2 sheets], WAG).

= Eragrostis tenuifolia (A.Rich.) Hochst. ex Steud., Syn. P1. Glumac. 1: 268 (1854). 
199. Poa unioloides Retz., Observ. Bot. 5: 19 (1788). - TYPE: India, J.G. König s.n. (lectotype LD [LD1289627], designated by Fischer, Bull. Misc. Inform. Kew 1932: 74 (1932); possible isolectotypes BM[2 sheets], C[3 sheets], LE).

= Eragrostis unioloides (Retz.) Nees ex Steud., Syn. Pl. Glumac. 1: 264 (1854).

200. Poa viscosa Retz., Observ. Bot. 4: 20 (1786-1787). - TYPE: J.G. König s.n. (lectotype LD [LD1289687], designated by Fischer, Bull. Misc. Inform. Kew 1932: 74 (1932); possible isolectotypes C[3 sheets]).

= Eragrostis tenella (L.) P.Beauv. ex Roem. \& Schult., Syst. Veg. 2: 576 (1817).

201. Pollinia ciliata Trin., Mém. Acad. Imp. Sci. St.-Pétersbourg, Sér. 6, Sci. Math. 2: 306 (1832). - TYPE: Nepal, N. Wallich s.n. (lectotype LE [Herb. Trinius 54.1], designated here; possible isolectotypes BM, C, CAL, E, G, K, P).

= Microstegium fasciculatum (L.) Henrard, Blumea 3: 453 (1940).

Notes. Trinius received his specimen from Hornemann in Copenhagen who in turn received it from fellow Dane, Nathaniel Wallich, probably while he was still in India, rather than from the dispersal of the East India Company Herbarium. The grasses were one of the families that were not sorted until after Wallich retired to London in 1846 (de Candolle \& Radcliffe-Smith, 1981). Material distributed under number 8815B may include duplicate collections of Trinius's specimen, but this cannot be known for certain.

202. Pollinia praemorsa Nees ex Steud., Syn. Pl. Glumac. 1: 409 (1854). - TYPE: Anon. (probably Zollinger), 'Java' (not traced).

= Polytrias indica (Houtt.) Veldkamp, Blumea 36: 180 (1991).

203. Rottboellia exaltata L.f., Suppl. P1. 114 (1782), nom. illeg., non L.f. (1779). - Manisuris exaltata Kuntze, Revis. Gen. Pl. 2: 779 (1891). - TYPE: C.P. Thunberg s.n. (lectotype LINN [Herb. Linn. 101.5], designated by Clayton, Kew Bull. 35: 817 (1981)).

= Rottboellia cochinchinensis (Lour.) Clayton, Kew Bull. 35: 817 (1981).

204. Rottboellia glandulosa Trin., Mém. Acad. Imp. Sci. St.-Pétersbourg, Sér. 6, Sci. Math. 2: 250 (1832). - TYPE: Java, Anon. s.n. (holotype LE [Herb. Trinius 113.1]). = Mnesithea glandulosa (Trin.) de Koning \& Sosef, Blumea 31: 290 (1986). 
205. Rottboellia repens G.Forst., Fl. Ins. Austr. 9 (1786), as 'Rottboella'. - TYPE: [unpublished illustration] Forster drawing 20 (lectotype BM, designated by Nicolson \& Fosberg, Forsters Bot. $2^{\text {nd }}$ Cook Exped. 234 (2004)).

= Lepturus repens (G.Forst.) R.Br., Prodr. 207 (1810).

206. Rottboellia sanguinea Retz., Observ. Bot. 3: 25 (1783). - TYPE: China, P.J. Bladh s.n. (lectotype LD [LD1224426], designated by Clayton \& Renvoize, F1. Trop. E. Africa, Gram. (Part 3): 756 (1982), or possible holotype).

= Schizachyrium sanguineum (Retz.) Alston in Trimen, Handb. Fl. Ceylon 6: 334 (1931).

207. Rottboellia striata Nees ex Steud. var. glabrior Hack. in A.DC. \& C.DC., Monogr. Phan. 6: 302 (1889). - TYPE: Singapore, October 1822, N. Wallich s.n. [EIC 8876] (lectotype K-W [K001131724], designated here; possible isolectotypes G [G00164612], K-W [K001131726]).

= Mnesithea glandulosa (Trin.) de Koning \& Sosef, Blumea 31: 290 (1986).

Notes. If Hackel had a specimen of this gathering in his collection it is not now present in W. We therefore designate a specimen from the Wallich Herbarium as lectotype as it is a good specimen and bears Wallich's original collection ticket.

208. Saccharum alopecurus Nees, Hooker's J. Bot. Kew Gard. Misc. 2: 100 (1850). - Saccharum negrosense Steud., Syn. P1. Glumac. 1: 407 (1854), nom. illeg. (superfl.). - Imperata ramosa Andersson, Öfvers. Förh. Kongl. Svenska Vetensk.-Akad. 12: 158 (1855), nom. illeg. (superfl.) - TYPE: Philippines, Island of Negros, H. Cuming 1801 (lectotype CGE (ex herb. Lindl.), designated here by Veldkamp; isolectotypes CGE (ex Mus. Henslow), E [E00393731], GOET[2 sheets], K, P, W).

= Imperata cylindrica (L.) Raeusch, Nomencl. Bot. 3: 10 (1797).

209. Saccharum arundinaceum Retz., Observ. Bot. 4: 14 (1786-1787). - TYPE: India, Pee Carumbo, J.G. König s.n. (lectotype LD [LD1227677], designated by Fischer, Bull. Misc. Inform. Kew 1932: 72 (1932); possible isolectotypes C[4 sheets]).

210. Saccharum confertum J.Presl in C.Presl, Reliq. Haenk. 1: 346 (1830). - TYPE: Philippines, Luzon, Sorsogon, T.P.X. Haenke s.n. (lectotype PR [sheet no. 612328], designated here by Veldkamp; isolectotype PR [sheet nos. 612329, 612330], US (fragment)).

=Imperata conferta (J.Presl) Ohwi, Bot Mag. (Tokyo) 55: 549 (1941). 
211. Saccharum fuscum Roxb., Fl. Ind. 1: 241 (1820). - TYPE: W. Roxburgh s.n. (lectotype K [K000943446], first step designated by Roberty, Boissiera 9: 367 (1960), second step designated here).

= Miscanthus fuscus (Roxb.) Benth., J. Linn. Soc., Bot. 19: 65 (1881).

Notes. Roberty referred to a Roxburgh gathering as type of Saccharum fuscum, but without indicating to which herbarium he was referring. There are Roxburgh specimens of this species in BM, BR and K. Only two sheets bear tickets with the original name in Roxburgh's hand - one from Forsyth's herbarium in K and one from the Roxburgh collection that was sold by the Linnean Society and is now in BR. The former is selected here as the Brussels' specimen is mounted in a somewhat condensed fashion making it more difficult to distinguish features.

212. Saccharum macilentum Chauv. ex Steud., Syn. Pl. Glumac. 1: 406 (1854). -TYPE: Ins. Waigiou [Waigeo], J.S.C. Dumont d'Urville s.n. (lectotype P [P03639080], designated here).

= Imperata conferta (J.Presl) Ohwi, Bot. Mag. (Tokyo) 55: 549 (1941).

Notes. The specimen referred to was annotated by Steudel but it is possible that he saw other material collected by Dumont d'Urville in Paris or elsewhere. We therefore designate the specimen as lectotype.

213. Saccharum paniceum Lam., Encycl. 1: 595 (1785). - TYPE: Sonnerat s.n. (holotype P-LA [IDC microfiche 6207: 686/20]).

= Pogonatherum crinitum (Thunb.) Kunth, Enum. P1. 1: 478 (1833).

214. Saccharum repens Willd., Sp. P1., ed. 4, 1: 322 (1797). - TYPE: Guinea [Ghana], P.E. Isert s.n. (lectotype B-W [B-W01499010], designated by Fosberg, Smithson. Contr. Bot. 47: 3 (1981); isolectotypes C, S).

= Melinis repens (Willd.) Zizka, Biblioth. Bot. 138: 55 (1988).

215. Saccharum ridleyi Hack., Oesterr. Bot. Z. 41: 6 (1891). - TYPE: Peninsular Malaysia, Pahang, Pekan, riverbank, May 1890, H.N. Ridley 1189 (lectotype W [1916-0034697], designated here by Veldkamp; isolectotypes K, SING, US (fragment)).

= Miscanthus fuscus (Roxb.) Benth., J. Linn. Soc., Bot. 19: 65 (1881). 
216. Saccharum spicatum L., Sp. P1. 1: 54 (1753). - TYPE: [published illustration] 'Tsjeria-kuren-pullu', Rheede, Hort. Malab. 12: t. 62 (1693) (lectotype designated by Clayton, Fl. Trop. E. Africa, Gram. (Part 2): 395 (1974)). Anon. s.n. (epitype LINN [Herb. Linn. 77.5], designated by Veldkamp \& Steenbergen, Austrobaileya 3 (1992) 610 , and corrected here by Veldkamp from lectotype).

= Perotis indica (L.) Kuntze, Rev. Gen. Pl. 2: 787 (1891).

217. Saccharum spontaneum L., Mant. P1. Altera 183 (1771). - TYPE: J.G. König s.n. (lectotype LINN [Herb. Linn. 77.1], designated by Cope, Fl. Pakistan 143: 263 (1982)).

218. Sacciolepis turgida Ridl., F1. Malay Penins. 5: 231 (1925). - TYPE: Peninsular Malaysia, Johore, Tana Merah Road, 1890, H.N. Ridley 1708 (lectotype SING [SING0054509], designated here by Veldkamp).

= Sacciolepis indica (L.) Chase, Proc. Biol. Soc. Washington 21: 8 (1908).

Notes. Gilliland (1964, p. 334), chose a Ridley specimen from Tanglin, Singapore, in $\mathrm{K}$ as the lectotype. This specimen cannot be found, so Veldkamp's replacement designation is made above.

219. Schizachyrium paradoxum Buse, Gram. 19 (Feb 1854); Buse, in Miq., P1. Jungh. 3: 359 (Aug 1854). - TYPE: Indonesia, Sumatra, Padang, F.W. Junghuhn s.n. (lectotype L [L0819911], first step designated by Jansen, Reinwardtia 2: 337 (1953), second step designated here; isolectotypes BRI, L [L0050060]).

= Schizachyrium brevifolium (Sw.) Nees in Mart., Fl. Bras. Enum. P1. 2: 332 (1829).

220. Schizachyrium semiberbe Nees in Mart., Fl. Bras. Enum. P1. 2: 336 (1829). - TYPE: Brazil, F. Sellow s.n. (lectotype B [B100383436], designated by Judziewicz, F1. Guianas, ser. A, Phanerogams 8: 579 (1990); isolectotypes BR[2 sheets], HAL, K, LE, MVFA (fragment), P, US, W[2 sheets]).

= Schizachyrium sanguineum (Retz.) Alston in Trimen, Handb. Fl. Ceylon 6: 334 (1931).

221. Setaria splendida Stapf, Fl. Trop. Afr. 9: 799 (1930). - TYPE: Sudan, Lado District, August 1916, T. Cartwright 18 (lectotype K [2 sheets] [K000281916 \& K000281915], designated by Morrone et al., Syst. Bot. Monogr. 96: 120 (2014)).

= Setaria sphacelata (Schumach.) Stapf \& C.E.Hubb., Fl. Trop. Afr. 9: 795 (1930). 
222. Sporobolus diandrus (Retz.) P.Beauv. var. major Buse, Gram. 3 (Feb 1854); Buse in Miq., Pl. Jungh. 3: 343 (Aug 1854). - TYPE: Indonesia, Java, Tjibogo, [June], F.W. Junghuhn s.n. (lectotype L [L0050184], designated by Baaijens \& Veldkamp, Blumea 35: 437 (1991)).

= Sporobolus indicus (L.) R.Br. var. major (Buse) Baaijens, Blumea 35: 437 (1991).

223. Stegosia cochinchinensis Lour., Fl. Cochinch. 51 (1790). - TYPE: J. Loureiro s.n. (lectotype BM [BM000513692], designated by Merrill, Trans. Amer. Philos. Soc. 25(2): 71 (1935)).

= Rottboellia cochinchinensis (Lour.) Clayton, Kew Bull. 35: 817 (1981).

224. Stipa arguens L., Sp. P1., ed. 2, 1: 117 (1762). - TYPE: J. Burman s.n. (lectotype LINN [Herb. Linn. 94.10], designated by Merrill, Interpr. Herb. Amboin. 89 (1917)).

= Themeda arguens (L.) Hack. in A.DC. \& C.DC., Monogr. Phan. 6: 657 (1889).

225. Thuarea sarmentosa Pers., Syn. P1. 1: 110 (1805). - TYPE: Madagascar, L.M.A. du Petit-Thouars s.n. (lectotype P [P02239717], designated here; possible isolectotype $\mathrm{P}[\mathrm{P} 02239716])$.

= Thuarea involuta (G.Forst.) R.Br. ex Sm., Cycl. 35, 2: unpaged (1 May 1817).

226. Tricholaena rosea Nees in Schauer, Cat. Sem. Hort. Vratisl. 1835: [3] (1836).

- TYPE: Drège 4319 (lectotype B [B100715446], designated here).

= Melinis repens (Willd.) Zizka, Biblioth. Bot. 138: 55 (1988).

227. Urochloa paspaloides J.Presl in C.Presl, Reliq. Haenk. 1: 318 (1830). - TYPE: [Philippines, Luzon, Sorsogon] T.P.X. Haenke s.n. (lectotype MO [MO-157629], designated by Lamson-Scribner, Rep. (Annual) Missouri Bot. Gard. 10: 36, 54 (1899); isolectotypes PR [sheet no. 194792], W[2 sheets]).

= Urochloa glumaris (Trin.) Veldkamp, Blumea 41: 420 (1996).

228. Vilfa berteroana Trin., Mém. Acad. Imp. Sci. Saint-Pétersbourg, Sér. 6, Sci. Math., Seconde Pt. Sci. Nat. 6(2): 100 (1840). - TYPE: Dominican Republic, Santo Domingo, C.G. Bertero s.n. (lectotype LE [Herb. Trinius 1682.1], designated by Baaijens \& Veldkamp, Blumea 53: 422 (1991); possible isolectotypes B, MO).

= Sporobolus indicus (L.) R.Br., Prodr. 1: 170 (1810). 
229. Zoysia japonica Steud., Syn. Pl. Glumac. 1: 414 (1854). - TYPE: Japan, P.F. von Siebold s.n. (lectotype L [L0050865], designated here by Veldkamp; possible isolectotypes L [L0050866], P [P00745709]).

230. Zoysia malaccensis Gand., Bull. Soc. Bot. France 64: 303 (1920). - TYPE: Singapore, H.N. Ridley s.n. (lectotype US [US00141793], designated here; isolectotype LY).

= Zoysia matrella (L.) Merr., Philipp. J. Sci., C 7: 230 (1912).

231. Zoysia matrella (L.) Merr. var. pacifica Goudswaard, Blumea 26: 172 (1980). - TYPE: Japan, Ryukyus, Yonakuni [Yonaguni] island, 20-30 October 1959, S. Hatusima 24127 (holotype L [L0050871]; isotype FU).

= Zoysia pacifica (Goudswaard) M.Hotta \& Kuroki, Acta Phytotax. Geobot. 45: 71 (1994).

232. Zoysia pungens Willd., Neue Schriften Ges. Naturf. Freunde Berlin 3: 441 (1801). - TYPE: D. Klein 3671 (lectotype B-W [B-W01514010], designated by Goudswaard, Blumea 26: 171 (1980); isolectotypes LE [Herb. Trinius 396.1, 4 excl. upper left], S [S-6-6510], US (fragment)).

=Zoysia matrella (L.) Merr., Philipp. J. Sci., C 7: 230 (1912).

ACKNOWLEDGEMENTS. Peter Hovenkamp (L) is thanked for locating and forwarding to us files left by JeF Veldkamp. The following are acknowledged for their valuable assistance with locating type material: Hans-Joachim Esser (M), Lauren Gardiner (CGE), Bazilah Ibrahim (SING), Laurence Loze (G), Misako Mishima (FU), Chiara Nepi (FI), Ranee Prakash (BM), Otakar Śída (PR), Robert Vogt (B). Henry Noltie (E) is thanked for confirming that a specimen in E is not a Koenig collection, and John McNeill (E) for discussions on lemon-grass nomenclature. The two reviewers are thanked for their comments.

\section{References}

Baas, P. \& Hovenkamp, P.H. (2018). In memoriam Jan-Frits Veldkamp (31 March 1941 - 12 November 2017). Blumea 63: 1-10.

Baldini, R.M. \& Longhi Wagner, H.-M. (2006). Poaceae Raddianae: an updated nomenclatural and taxonomical evaluation of G. Raddi's Brazilian Poaceae. Taxon 55: 469-482.

Bor, N.L. (1955). Notes on Asiatic grasses: XX. Indian grasses in Roth's herbarium. Kew Bull. 9: 545-548.

Chase, A. \& Niles, C.D. (1962). Index to grass species. Volume 2, E-Pan. Boston: G. K. Hall $\&$ Co. 
de Candolle, R. \& Radcliffe-Smith, A. (1981). Nathaniel Wallich, MD, PhD, FRS, FLS, FRGS, (1786-1854) and the Herbarium of the Honourable East India Company, and their relation to the de Candolles of Geneva and the Great Prodromus. Bot. J. Linn. Soc. 83: 325-348.

de Koning, R. \& Sosef, M.S.M. (1985). The Malesian species of Paspalum L. (Gramineae). Blumea 30: 279-318.

Duistermaat, H. (2005). Field guide to the grasses of Singapore (excluding the bamboos). Gard. Bull. Singapore 57(Suppl.): 1-177.

Forman, L. (1997). Notes concerning the typification of William Roxburgh's species of phanerogams. Kew Bull. 52: 513-534.

Gilliland, H.B. (1964). Further notes on the grasses of the Malay Peninsula I. Gard. Bull., Singapore 20: 313-314.

Hitchcock, A.S. (1908). Types of American grasses: a study of the American species of grasses described by Linnaeus, Gronovius, Sloane, Swartz, and Michaux. Contr. U.S. Natl. Herb. 12: 113-158.

Kneucker, A. (1915). Bemerkungen zu den „Gramineae exsiccatae“. 27.—32. Lieferung. 1914/15. Allg. Bot. Z. Syst. 1914: 142-146.

Koch, S.D. (1974). The Eragrostis pectinacea-pilosa complex in North and Central America (Gramineae: Eragrostoideae). Illinois Biol. Monogr. 48: 1-74.

McNeill, J. (2014). Holotype specimens and type citations: general issues. Taxon 63: $1112-1113$.

Nicolson, D.H. \& Fosberg, F.R. (2004). The Forsters and the botany of the second Cook Expedition (1772-1775). Ruggell: A. R. G. Gantner Verlag KG [Regnum Veg. vol. 139].

Noltie, H.J. (2005). The botany of Robert Wight. Ruggell: A. R. G. Gantner Verlag KG [Regnum Veg. vol. 145].

Noltie, H.J. (2010). A commentary on the new taxa described in The Botany of Captain Beechey's Voyage by W. J. Hooker and G. A. Walker-Arnott. Edinburgh: Royal Botanic Garden Edinburgh.

Roberty, G.E. (1960). Monographie systématique des Andropogonées du globe. Boissiera 9: $1-455$.

Stafleu, F.A. \& Cowan, R.S. (1986). Taxonomic literature. Vol. vi Sti-Vuy. Utrecht: Bohn, Scheltema \& Holkema [Regnum Veg. vol. 115].

Stapf, O. (1906). The oil-grasses of India and Ceylon. (Cymbopogon, Vetiveria and Andropogon spp.). Bull. Misc. Inform. Kew 1906: 297-363.

Stearn, W.T. \& Williams, L.H.J. (1957). Martin's French Guiana plants and Rudge's "Plantarum Guianae rariorum icones". Bull. Jardin Bot. Bruxelles 27: 243-265.

Traiperm, P., Boonkerd, T., Chantaranothai, P. \& Simpson, D.A. (2012). Notes on the genus Ischaemum (Poaceae). Kew Bull. 57: 75-79.

Turland, N.J., Wiersema, J.H., Barrie, F.R., Greuter, W., Hawksworth, D.L., Herendeen, P.S., Knapp, S., Kusber, W.H., Li, D.Z., Marhold, K., May, T.W., McNeill, J., Monro, A.M., Prado, J., Price, M.J. \& Smith, G.F. (eds) (2018). International Code of Nomenclature for algae, fungi, and plants (Shenzhen Code) adopted by the Nineteenth International Botanical Congress Shenzhen, China, July 2017. Regnum Vegetabile 159. Glashütten: Koeltz Botanical Books.

van der Zon, A.P.M. (1992). Graminées du Cameroun. Vol. 2, Flore. Wageningen Agr. Univ. Pap. 92-1: 1-557.

van Eck-Borsboom, M.H.J. (1980). A revision of Eriachne R.Br. (Gramineae) in Asia and Malesia. Blumea 26: 127-138. 
van Steenis-Kruseman, M.J. (1950). Malaysian plant collectors and collections: being a cyclopaedia of botanical exploration in Malaysia and a guide to the concerned literature up to the year 1950. In: van Steenis, C.G.G.J. (ed.) Flora Malesiana, ser. 1, Seed Plants, vol. 1., pp. 1-606. Jakarta: Noordhoff-Kolff.

Veldkamp, J.F. (1973). A revision of Digitaria Haller (Gramineae) in Malesia. Blumea 21: $1-80$.

Veldkamp, J.F. (1994). Miscellaneous notes on Southeast Asian Gramineae. IX Setaria and Paspalidium. Blumea 39: 373-384. 